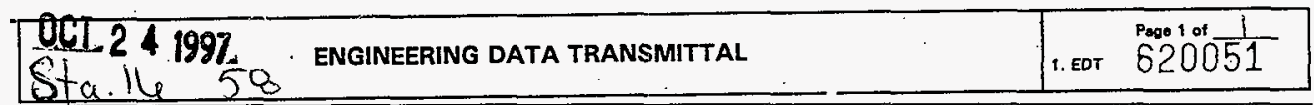

\begin{tabular}{|c|c|c|}
\hline $\begin{array}{l}\text { 2. To: (Receiving Organization) } \\
\text { See Distribution List }\end{array}$ & $\begin{array}{l}\text { 3. From: (originating Organization) } \\
\text { West Tank Farm Transition } \\
\text { Project Engineering }\end{array}$ & $\begin{array}{r}\text { 4. Related EDT No.: } \\
\text { N/A }\end{array}$ \\
\hline $\begin{array}{l}\text { 5. Proj./Prog./Dept./Div.: } \\
\text { HMT/DACS/Engr/74800 }\end{array}$ & $\begin{array}{l}\text { 6. Design. Authority/ Design Agent/Cog. } \\
\text { Engr:: } \\
\text { W.G. Brown/G.J. Gauck /N/W6B }\end{array}$ & $\begin{array}{l}\text { 7. Purchase Order No.: } \\
\qquad N / A\end{array}$ \\
\hline
\end{tabular}

8. Originator Remarks:

This document provides evaluation information to justify a Windows based Human Machine Interface (HMI) replacement to the existing DOS based Iconics HMI currently used in the Data Acquisition and Control System (DACS) used to mitigate flammable gas at 241-SY-101.

11. Receiver Renarks: 11A. Design Baseline Document? [] Yes [X] No The report conclades with the recoumendafion of Intellution's FIX software to be used at DACS Grailer (ma-46/) to provide a Windows basel HMI to replace the existing DOS based Iconics HMI.

9. Equip:/Component No.: $\mathrm{N} / \mathrm{A}$

10. System/Bldg./Facility: $241-S Y-101$

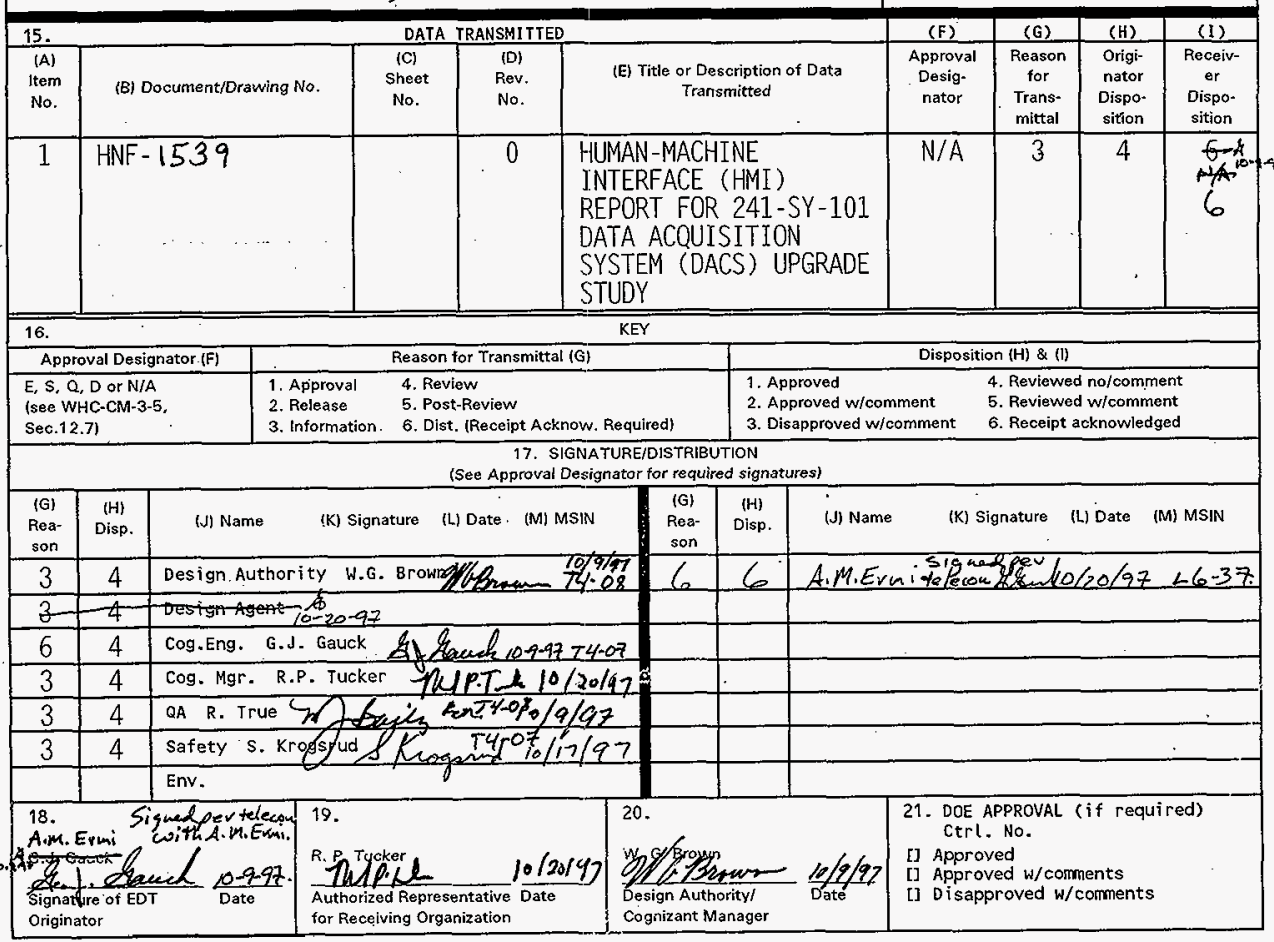

BD-7400-172-2(05/96) GEF097 


\section{HUMAN-MACHINE INTERFACE (HMI) REPORT FOR 241-SY-101 DATA ACQUISITION SYSTEM (DACS) UPGRADE STUDY}

R.W Truitt

PLC'S Plus, Richland. WA 99352 SESC

U.S. Department of Energy Contract DE-AC06-96RL13200

EDT/ECN: 620051

Org Code: 74800

B\&R Code: EW3120072
UC: UC-2030

Charge Code: N1W6B

Total Pages: 6667

time $10 / \mathrm{sen} / 77$

Key Words: HMI, System Architecture, Operating System, Networking, Configuration, Development, Database, Hardware, Software, Scripting

Abstract: This report provides an independent evaluation of information for a Windows based Human Machine Interface (HMI) to replace the existing DOS based Iconics HMI currently used in the Data Acquisition and Control System (DACS) used at 241-SY-101

Per General Counsel documents that list products for Conceptual Design or Procurement have been given a waiver for trademark call out.

TRADEMARK DISCLAIMER. Reference herein to any specific comercial product, process, or service by trade name, trademark, manufacturer, or otherwise, does not necessarily constitute or imply its endorsement, recommendation, or favoring by the United States Government or any agency thereof or its contractors or subcontractors.

Printed in the United States of America. To obtain copies of this document, contact: Document Control Services, P.0. Box 950, Mailstop H6-08, Richland WA 99352, Phone (509) 372-2420; Fax (509) 376-4989.
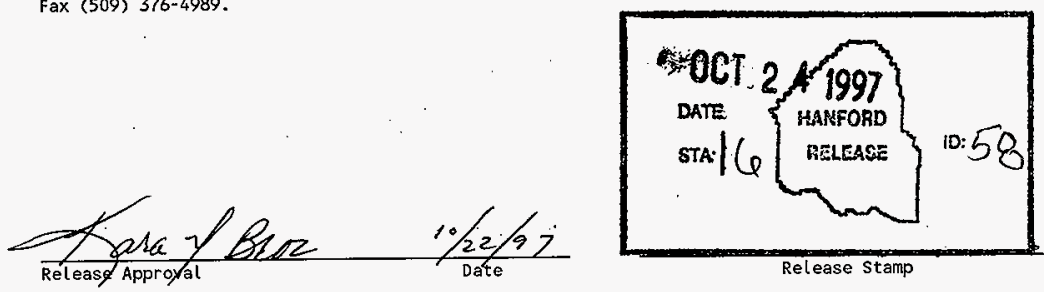

Release Stamp 


\title{
HUMAN-MACHINE INTERFACE (HMI) REPORT FOR 241-SY-101 DATA ACQUISITION SYSTEM (DACS) UPGRADE STUDY
}

\author{
Prepared by: \\ PLCs Plus \\ Richland, WA
}

August 1997 


\section{TABLE OF CONTENTS}

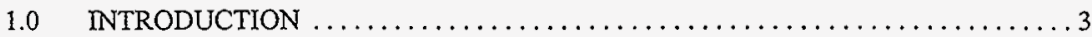

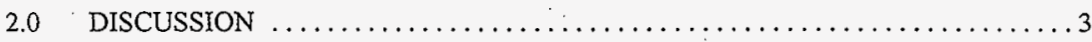

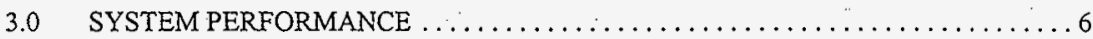

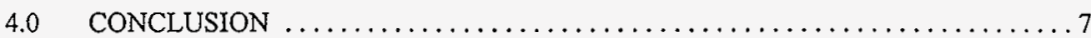

$5.0 \quad$ PRODUCT DESCRIPTIONS $\ldots \ldots \ldots \ldots \ldots \ldots \ldots \ldots \ldots \ldots \ldots \ldots \ldots \ldots$

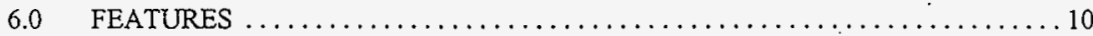

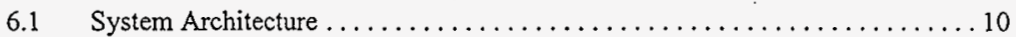

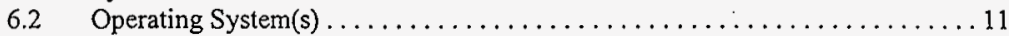

6.3 Open/Scalable/Flexibility/Compatibility ..................... 12

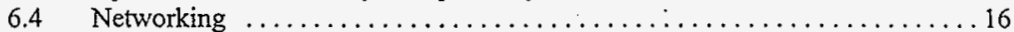

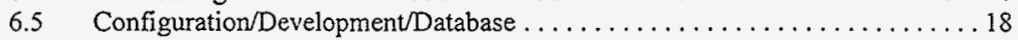

6.6 Hardware/Sofware - System Specifications ................... 20

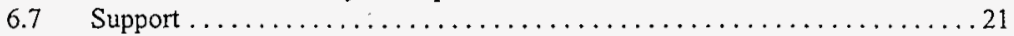

$6.8 \quad$ Scripting $\ldots \ldots \ldots \ldots \ldots \ldots \ldots \ldots \ldots \ldots \ldots \ldots \ldots \ldots, 22$

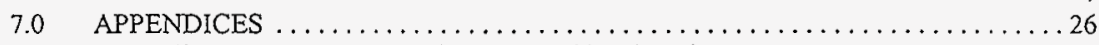

Appendix A: $\quad$ Reprint from Control Engineering, February 1997

Appendix B: $\quad$ Reprint from Managing Automation, June 1997

Appendix C: $\quad$ Reprint from Manufacturing_Systems, July 1996

Appendix D: $\quad$ PLCs PLUS Internal Survey

Appendix E: $\quad$ Competitive Review - Paragon TNT

Appendix F: $\quad$ Reprint from InTech, May 1996

Appendix G: $\quad$ Reprint from Design Engineering, June 1996

Appendix H: $\quad$ Reprint from Measurements and Control, April 1996

Appendix I: . Reprint from Computerworld, May 1996

Appendix J: $\quad$ Reprint from Maintenance Technology, May 1996 


\subsection{INTRODUCTION}

PLCs Plus was tasked with the responsibility of producing a Human Machine Interface (HMI) report. The purpose of the report is to provide evaluation information for a Windows based HMI to replace the existing DOS based Iconics HMI currently used in the Data Acquisition And Control System (DACS) used at 241-SY-101.

A fundamental reason for this evaluation is because of the difficulty of maintaining the system with obsolete, unsupported software. The DACS uses a software operator interface (Genesis for DOS HMI) that is no longer supported by its manufacturer, Iconics. In addition to its obsolescence, it is complex and difficult to train additional personnel on. The FY 1997 budget allocated \$40K for phase 1 of a software/hardware upgrade that would have allowed the old DOS based system to be replaced by a current Windows based system. Unfortunately, budget constraints during FY 1997 has prompted deferral of the upgrade.

The upgrade needs to be performed at the earliest possible time, before other failures render the system useless. Once completed, the upgrade could alleviate other concerns: spare pump software may be able to be incorporated into the same software as the existing pump, thereby eliminating the paraliel path dilemma; and the newer, less complex software should expedite training of future personnel, and in the process, require that less technical time be required to maintain the system.

\subsection{DISCUSSION}

Information presented in this HMI report has been compiled from several sources. Each of the HMI suppliers' internet web sites and sales literature were used in gathering pertinent data regarding these suppliers and the systems they offer.

PLCs Plus does not assume any responsibility for the validity of the information provided by suppliers, as only the suppliers can verify the accuracy of their statements. Verification would best be accomplished by witnessing demonstrations of those systems that seem applicable to the needs of the 241-SY-101 project.

Other sources of information for data used in this report are: Managing Automation (June 1997), Control Engineering (February 1997), Manufacturing Systems (July 1996), and the engineering staff of PLCs Plus. 
The following topics were selected because of the estimated impact with regard to a decision for selection of an HMI to be used as a possible upgrade for the current system used at DACS, controlling the Hydrogen Mitigation mixer pump in tank 241-SY-101: Product Descriptions, Features - System Architecture, Operating System(s), Open/Scalable/Flexibility/Compatibility, Networking, Configuration/Development/ Database, Hardware/Software - System Specifications, Support, and Scripting.

Items not covered in this report, since several of the products capabilities were judged to be relatively equal, were: Graphics, Drivers, Trends/Reports/History, Alarms, Security, Wizards/Dynamos, Optimization/Polling.

Four suppliers were selected for consideration: Iconics-Genesis For Windows, WonderwareInTouch, Intellution-FLX, and $\mathrm{Ci}$ Technologies-Citect. The suppliers chosen for review in this report were based on the experience of PLCs Plus, availability of the products for evaluation, and various demonstrations presented by the suppliers. The order of presentation of the products was selected randomly as follows: Iconics, Wonderware, Intellution, and Citect. Product discussions throughout this report were kept in this order to help simplify reading the report.

Also, included in this report are four surveys, three from industry publications and one that was conducted as an internal survey by the staff of PLCs Plus. Brief results are as follows:

The Control Engineering survey is an alphabetical listing of approximately 50 companies' Supervisory Control And Data Acquisition (SCADA) software. There are two significant categories: Operating System and Features. The survey shows only Iconics having exceptions compared to the other three. It is indicated that Iconics is still reliant upon a DOS based Real-Time Server and is not capable of Multi-Channel Signal Conditioning. All of the other suppliers provide MultiChannel Signal Conditioning. (Appendix A provides complete results of the survey.)

Managing Automation's, "Hot 100 Manufacturing Software Companies". listing is based on a composite of size, market dominance, and technology. This is an alphabetical listing, rather than a ranked list of their "choice of significant software vendors." There are four items listed for each of the companies covered in this report: Location, Product, Application, and Outlook. (Appendix B provides - complete results of the survey.) 
From Manufacturing Systems "Top 50" survey, the rankings are based solely on revenue derived from manufacturing software in calendar year 1995. Some key comments taken from the listing are:

Iconics - did not make the Top 50 listing.

Wonderware - "appeared to hit rough waters" and was forced to "grow-up" after experiencing entrepreneurial growth. It was stated, "not so much a company in dire straits, but one working to establish a new identity."

Intellution - "continues to chum out leading-edge process-automation and control software, and manufacturing companies around the world keep gobbling it up." Current successes are attributed to "the market's rapid adoption of 32-bit computing environments. Thirtytwo bit computing is a synonym for Windows NT and Windows 95 . Intellution began building its products for those environments in 1993, and it appears customer demand for those systems finally has materialized." Intellution received Microsoft's Leonardo Medal for Technical Innovations in Manufacturing. It was stated, this "solidifies our leadership role in the industrial automation software market." (Appendix $\mathrm{C}$ contains the complete results of the survey.)

An evaluation copy of Citect was not available at the time of the PLCs Plus internal survey, so only Iconics, Wonderware, and Intellution software were considered. Thirteen categories were defined with a weighted ranking assigned to each, 5=BEST to 1 =WORST. (Appendix D lists the complete results of the survey.) 
Table A lists the strengths and weaknesses of each product, according to the PLCs Plus staff:

\section{Table A}

\begin{tabular}{|l|l|l|}
\hline PRODUCT & STRENGTHS & WEAKNESSES \\
\hline Iconics & Tools, Scripting (VBA) & $\begin{array}{l}\text { Operating System, } \\
\text { Difficult to Learn, } \\
\text { Reliability }\end{array}$ \\
\hline Wonderware & Technical Support & $\begin{array}{l}\text { Operating System, } \\
\text { Drivers, Networking }\end{array}$ \\
\hline Intellution & $\begin{array}{l}\text { Operating System, } \\
\text { Drivers, Networking, } \\
\text { Reliability }\end{array}$ & None Identified \\
\hline
\end{tabular}

Microsoft Windows is the dominant operating system for PC-based systems. On the plant floor, Windows NT 4.0 will solidify that position as more manufacturing companies adopt it as their operating system platform. Vendors with Windows 3.X or DOS applications will be forced into 32bit development leaving 16-bit development all but "dead." The scaleability, flexibility, and power of NT will continue to attract users and vendors providing low-cost and high-performance engineering solutions.

With development of an OLE-based data exchange standard as their focus, five companies - FisherRosemount Systems, Intellution, Intuitive Technology, Opto-22, and Rockwell Software -- along with OLE consultants from Microsoft, formed the OLE for Process Control (OPC) Task Force. This standard will be instrumental in overcoming challenges of sharing information among intelligent devices on the plant floor.

\subsection{SYSTEM PERFORMANCE}

Performance is an often ignored element in the evaluation of HMI systems. However, our recommendation would be to limit the choices to one or two products then perform tests, including the following items:

- Data acquisition speed.

- Data processing speeds (alarms, calculations, etc.).

- Data presentation speeds (graphics).

- Data storage speeds (historical archiving).

- System responsiveness to user requests.

$$
\begin{gathered}
\text { HNF-1539, Rev. } 0 \\
\text { Page } 6
\end{gathered}
$$


Since data acquisition is the data source for most other HMI tasks, it is often acknowledged as one of the key system performance features and because of this the performance of device drivers is very important. Since device drivers are the interface gateways to the rest of the system, these drivers limit the overall performance of HMI software and control the updating capabilities of the other HMI tasks.

Repeatable testing with a number of programmable controllers and with a number of HMI systems has been performed. Consistently, DLL drivers outperform DDE drivers by a factor of 10 and the end-user is dependant on the device-driver performance. The update efficiency of graphics, trends, and other functions depends on the device driver, and directly controls the presentation of timely information used to make critical, informed decisions.

\subsection{CONCLUSION}

The evidence as indicated from the various reference sources used in this report leads to the recommendation of Intellution's FIX software to be used at DACS. The data in Control Engineering's survey reveals there are serious drawbacks of a DOS based system, such as Iconics Genesis. (Some of the problems include limited vendor support, 16-bit portability, training difficulties, and more.) Manufacturing System's "Top 50" rankings lists favorably the 32-bit Windows NT based "leading edge" software from Intellution. Also, the DACS computers are "highly" networked, with networking being another strong point of Intellution.

Intellution has become more prevelant on the Hanford Site. There are several projects currently using Intellution software: PUREX - SAMCONS, W211, W259 T-Plant, and Spent Nuclear Fuel Canister Storage Building and Cold Vacuum Drying Facility.

This report is intended to give an overview of these products for possible use at DACS. Hopefully, this information will prove useful as a tool in making an informed decision related to these product technologies and associated vendors. 
Note: The information presented in the following pages is vendor supplied.

\subsection{PRODUCT DESCRIPTIONS}

\section{Ieonics}

The complete Automation Tool Suite for MMI, SCADA and Control! See for yourself how the Project Manager coordinates the operation of GFW's modular components and how GEN-NET is used to connect GENESIS for Windows and GENESIS Control Series (DOS) workstations. Other features include Dynamic Data Exchange (DDE), and VBA Compatible Scripting. For additional information see Specifications and Accessories.

Introducing GENESIS for Windows the first complete software Automation Tool Suite for PC-based Operator Interface (MMI) and Supervisory Control (SCADA). GENESIS for Windows (GFW) features a suite of independent 'Best-In-Class' applications for graphics, trending and data logging, alarm management, VO scanning, control logic, and more. These are just a few of the features that make GENESIS for Windows the best choice for any application.

GENESIS for Windows is a total software solution for factory automation and process management. GFW is a modular system, made up of fully independent client and server applications. Assemble your own custom MMLSCADA system by combining the GENESIS for Windows modular components with any other Windows software.

\section{Wonderware InTouch}

Wonderware pioneered the use of Microsofta Windows on the factory floor in 1989, with the introduction of our revolutionary InTouch ${ }^{\mathrm{TM}}$ man-machine interface (MMI) software. Since then, Wonderware has rapidly become the world leader in industrial automation solutions, offering the Wonderware Factory Suite ${ }^{\mathrm{TM}}$ of products that span the enterprise, from the factory floor to the executive offices -- from supervisory control and data acquisition (SCADA) applications to production management and resource tracking, flexible batch management, expert machine diagnostics, and connectivity products to link them to control devices and computing networks. 
Wonderware InTouch is an object-oriented, graphical man-machine interface (MMI) application generator for industrial automation, process control and supervisory monitoring applications. A core module of Factory Suite, InTouch allows engineers to readily create, on a computer display screen, graphical representations of the physical devices (gauges, meters, knobs, switches, etc.) used to control equipment in a factory or process control system - and to animate them with a wide range of properties to replicate the real process. Version 6.0 for Windows NT 4.0 includes several new distributed alarm handling, distributed historical data, dynamic resolution conversion and remote application development and maintenance for use in large PC-based networks. Enhancement modules include Recipe Manager, Statistical Process Control (SPC) and SQL Access. Included with InTouch is Factory Focus ${ }^{\mathrm{TM}}$, a view-only node that gives managers and office workers a low-cost and convenient way to monitor plant floor operations from their desktop anywhere within a company. InTouch 6.0 also contains the Wonderware Productivity Pack, a handy software tool that helps users to develop custom wizards. For Windows NT users, the Productivity Pack has more than 2,000 wizards that make application development easier than ever before.

\section{Intellution}

For more than 15 years, Intellution has been setting the industry standard for easy, powerful, and reliable automation solutions for manufacturers across the globe.

The \#1 Automation Software for Windows 95 and Windows NT. A True 32-bit design - Intellution's proven 32-bit FIX ${ }^{\mathrm{TM}}$ automation software is the only automation solution that takes full advantage of all the powerful capabilities of Windows 95 and Windows NT. With more 32-bit installations than all other automation software vendors combined, only Intellution has the experience and technology to solve your most difficult manufacturing challenges, now and in the future. Unlock the power, performance and security of Windows 95 and Windows NT with the proven 32-bit performance of Intellution's 32-bit FIX automation software. 32-bit processing is the future of industrial automation, and it's here today with Intellution's 32-bit FIX software for Windows 95 and Windows NT.

Intellution's FIX family of products for MMI and SCADA applications is consistently rated number one by industry analysts, integrators and end-users alike. The FIX is industry's top choice because it enables users to quickly and easily build a powerful window into their manufacturing operation.

\section{Citect}

\section{Welcome to Citect for Windows!}

You can configure a Citect monitoring and control system to suit any industrial application. And because Citect has been designed with flexibility in mind, you can design a system to suit your exact requirements. 
Citect suits both small and large applications. Because it is flexible, Citect will keep pace with your plant and information requirements as they change and expand.

JO Optimization

- Operates on a demand basis $\bullet$ Supersedes the conventional predefined scan method $\bullet$ Automatic optimization of block data requests to PLCs and DCs $\bullet$ Automatic rationalization of network wide user data requests $\bullet$ Maximization of control vendors highway bandwidth

Applications Language (Cicode)

-Fully pre-emptive multi-threaded language $\bullet$ Client Server, Remote Procedure Calls (RPC) $\bullet$ User Written Functions $\bullet$ Indirect Read and Write of tags $\bullet$ Functions are re-usable, modular and transportable throughout the system $\bullet$ Full mathematical and Boolean functions $\bullet$ Full string manipulation $\bullet$ If-then-else, For-Do, While-Do, Conditional Executors $\bullet$ Over 500 standard Cicode functions provided $\bullet$ Designed especially for plant monitoring and control applications

Citect is easy to learn and use. Features such as templates, genies, and wizards reduce the time and effort required to configure your Citect system, as well as maximize its performance.

\subsection{FEATURES}

\subsection{System Architecture}

\section{Iconics}

Information not available.

\section{Wonderware}

Information not available.

\section{Intellution}

FIX Automation Software Features:

- Distributed, Client/Server Architecture

- Intuitive Man-Machine Interface

- $100 \%$ Data Integrity

- Real-Time Process Monitoring

- Supervisory Control and Data Acquisition

$$
\begin{gathered}
\text { HNF }-1539 \text {, Rev } 0 . \\
P=-\infty
\end{gathered}
$$


- SQL/ODBC Relational Database Connectivity

- Alarming and Alarm Management

- Comprehensive, Accurate Reporting

- Real-Time and Historical Trending

- Statistical Process Control

- $\quad$ MMI and SCADA Solutions for All Size Applications

\section{Citect}

Systems Architecture

- Client Server Distributed Processing

- Open architecture design

- Imbedded real-time multi-tasking kernel

- Centralized alarm, trend and report processing

- Automatic update of configuration across a network

- Concurrent support of multiple display nodes

- Access any tag and or any data from any node

- Scalable architecture allows expansion without configuration changes

- Single Database configuration source for Large Networked Systems

Fault Tolerance

- Automatic change over and recovery

- Full hot standby. supported

- Supports primary and secondary equipment configurations

- Intelligent redundancy allows secondary equipment to contribute to processing load

- Dual Networks for full LAN Redundancy

- No configuration required may be enable via single check box

- Redundant writes to PLCs with no programming

- Redundant File Server Support

\subsection{Operating System(s)}

Iconics

DOS/Windows 3.X/95/NT 


\section{Wonderware}

Windows 3.X/95/NT

Wonderware continues to lead the industry in implementation of automation solutions based on Microsoft Windows $95^{\mathrm{TM}}$ and Windows NT ${ }^{\mathrm{rM}}$ operating systems as well as on the Microsoft BackOffice suite of server solutions. The Wonderware InTrack ${ }^{\mathrm{TM}}$ production management system, in fact, is the first -- and still the only -- industrial automation software system certified for use on Microsoft BackOffice.

\section{Intellution}

\section{Windows 3.X/95/NT}

FIX for Windows 95 and Windows NT is a true 32-bit software application written to Microsoft's Win-32 specification. This means that, unlike 16-bit software systems, FIX takes full advantage of all the powerful capabilities of Microsoft's Windows 95 and Window NT operating systems, including pre-emptive multitasking, multithreading, plug and play hardware compatibility, symmetric multiprocessing and much more.

The first automation software vendor to introduce a 32-bit application for the Windows NT platform. Intellution today has more 32-bit installations than all other automation software vendors combined.

\section{Citect}

Windows $3 . X / 95 / N T$

\subsection{Open/Scalable/Flexibility/Compatibility}

\section{Ietonies}

Recognized Standards

With today's fast changing technology, GENESIS for Windows is designed to stay current by adopting widely recognized standards. Exchange data with other Windows applications using Windows DDE and OLE. Enjoy direct database connectivity using ODBC and SQL. Distribute realtime data around the plant, or around the world, using TCP/IP networking. Customize the system with easy to leain Visual Basic scripting commands. Standards save engineering time and protect your valuable software investment. 
- Over 250 interfaces to PLCs, Loop Controllers, and V/O Systems for maximum industrial connectivity $\bullet$ Distributed client/server architecture for expandable plant wide networking $\bullet$ Powerful DDE, OLE, and direct DLL communication capacity that offers full connectivity to other applications

GEN-RTU is the Remote Terminal Unit option used for executing periodic communications with up to 120 remote $\mathrm{I} / \mathrm{O}$ devices via telephone modems. This provides a highly flexible, low cost solution for PC-based distributed data acquisition systems. Types of polling include sequential, periodic, and on-demand. Up to 300 telephone numbers can be configured and can be changed on the fly. Use this option whenever it is impractical to maintain continuous modem communications with remote $\mathrm{V} / \mathrm{O}$.

GEN-TEXT is an ASCII text messaging driver that provides a general purpose interface for sending predefined ASCII messages to a serial port based on logic within the RTS. Up to 1024 different messages can be defined. Use this option whenever custom ASCII text messages need to be sent to printers, telephone pagers, modems, or any other device that can be connected to COM 1-4. This option counts as one of the six RTS device drivers.

\section{ODBC Configuration Tools}

An UO Server consists of two parts-Configuration and Runtime. The configuration utility uses an ODBC compliant database to define the $\mathrm{VO}$ "points." This might include the tag name, point type (e.g., analog or digital), the memory location of the desired data, scaling parameters, alarm information, and so on. Separate screens configure the communication ports and addressing information of networked devices. The use of Microsoft standard ODBC technology means that the configuration information can be accessed, imported, or exported to a variety of database and spreadsheet programs. This makes it possible to transfer information from PLC programming software directly into the I/O Server configuration.

\section{Fast Runtime Engine}

The I/O Server Runtime engine performs the actual communication functions to the field devices. It is optimized to maintain a real-time database of only the I/O data and alarm information. Calculations and logic must be provided by other applications. This design helps the VO Server runtime use the minimum system resources for best performance. Multiple I/O Servers can be running simultaneously. 


\section{Large I/O Capacity}

Each $\mathrm{V} / \mathrm{O}$ point, or tag, can be configured for continuous update or scan-on-demand. Continuous updates are used for points which are always required, or which must be scanned for alarm conditions. With scan-on-demand, only those points being requested by the client applications are automatically updated. This method allows the I/O Server to simultaneously manage thousands of data points. Most applications use a combination of the two methods.

\section{Development Tool Kit}

A tool kit is available for users who want to create their own custom I/O Servers. The I/O Server developer needs only to define the configuration GUI and the Runtime communication protocol section. The I/O Server Tool Kit will do the rest.

\section{Wonderware}

\section{NetDDE}

Using Wonderware's NetDDE, included with InTouch, users benefit from DDE connectivity between applications on different nodes over the network. Connectivity support is provided between Windows, VMS, and UNIX operating environments over a range of network protocols such as NetBIOS, TCP/TP, and DecNET, plus serial port communication. NetDDE enables the workstation to be a virtual gateway between network protocols.

\section{Dynamic Tag Referencing}

InTouch has many NEW distributed features. Dynamic Referencing lets users change database references to input/output tags at runtime. This means that users can change data references on the fly for PLC addresses, Excel spreadsheet cells, Dynamic Data Exchange (DDE) references, and the like. Using this exciting new capability, operators can use a single tag to 'view' any cell in a spreadsheet, for example. Coupled with Wonderware's existing ability to dynamically switch between I/O servers or other DDE-aware applications, Dynamic Referencing provides tremendous application flexibility. Distributed Alarming supports multiple alarm servers or 'providers' simultaneously, which gives operators the ability to view alarm information from multiple remote locations at the same time. The new distributed alarm functions let users implement 'point-and-click' alarm acknowledgement, alarm scroll bars and many other new features for networked use. The new Distributed Historical Trending system allows users to dynamically specify different historical file data sources for each of the pens on a trend chart. Since InTouch permits the use of up to eight pens per trend chart, users can have an unprecedented amount of historical cata available for viewing at any given time. Remote Development features have been added to accommodate large multi-node installations. These include application updating of all nodes on a network, either automatically in a time-based fashion or in response to operator triggers or application events.

$$
\begin{gathered}
H N F-1539, \text { Reu. } \\
\text { p.an } 14
\end{gathered}
$$




\section{DDE I/O Servers}

DDE y/O Servers are input/output drivers that use Microsoft's Dynamic Data Exchange (DDE) protocol to provide seamless data sharing between Wonderware InTouch and programmable logic controllers (PLCs) or other control devices. The servers' use of DDE also allows the exchange of data with any DDE-aware Windows programs. Wonderware offers hundreds of DDE Servers, as well as a DDE Server Toolkit that lets users develop their own servers.

Professional Developers Kit (PDK)

The Wonderware InTouch PDK, available on CD-ROM, is a comprehensive product bundle consisting of Wonderware InTouch Standard for Windows, Windows 95 and a full 32-bit version for Windows NT. It includes all of Wonderware's powerful options: SPC, SQL Access, Recipe, NetDDE, all the L/O Servers, and documentation for all bundled software. The PDK is targeted at System Integrators and other power users.

\section{SQL Access}

Wonderware's SQL Access option provides InTouch users with access to virtually all of the large database programs, including Microsoft SQL Server, ORACLE, SyBase, dBase, and others that support the Open Data Base Connectivity (ODBC) standard.

\section{Extensibility Toolkit}

This software package allows users to customize and extend the capabilities of InTouch according to their specific needs. The Toolkit includes the Wizard Development Kit, the Script Enhancement $\mathrm{Kit}$ and the IDEA Toolkit. Users with " $\mathrm{C}$ " programming competence can create their own Wizards and unique script functions and include them as part of their development system. They can also tap into the power of Visual Basic, FORTRAN, Pascal, etc., for additional development of visual elements, forms, database access and manipulation.

\section{DDE I/O Servers}

A wide range of $\mathrm{W} O \mathrm{O}$ servers are available from Wonderware as well as third-party developers for hundreds of the most popular control devices, including Allen-Bradley, Siemens, Modicon, Opto 22, Square D and more. All Wonderware servers provide standard DDE communication to any Windows/DDE application as well as "fastDDE" for communication with Wonderware products. Wonderware also offers a DDE Server Toolkit that lets users develop new or proprietary DDE servers.

$$
\begin{gathered}
\text { HNF }-1539, \operatorname{Rev} .0 \\
\text { Page is }
\end{gathered}
$$




\section{Intellution}

- Open, Scalable and Compatible -- Expanding your FIX MMI system into a multi-node networked SCADA application is as easy as plugging a PC into your network. $\bullet$ Distributed, Client/Server Architecture -- All FIX products are designed for both stand-alone and networked applications. With our distributed client/server architecture, you can instantly share data across the enterprise, for faster, better-informed business decisions. - The Strategic Choice for Windows Platforms -- Because of Intellution's partnership with Microsoft and our commitment to developing high-performance solutions for Microsoft operating systems, your purchase of Intellution software is as safe and smart investment, for today and the future.

\section{Citect}

Citect systems network in the true spirit of Scalable Control Systems. Processing tasks can be distributed, multiple LANS added and redundancy built in - without replacing any hardware or software. File Server functions can be split into multiple nodes for brilliant performance in even the largest systems.

Access to Data

- Direct, real-time access to data by any network user $\bullet$ Third-party access to real-time data ie Access and excel $\bullet$ Network DDE including: Read, Write and Execute.

Database

$\bullet \mathrm{ODBC}$ driver support $\bullet$ Direct SQL commands $\bullet$ Built-in dBase file access

Protocol Compatibility

- DDE servers supported $\bullet$ Drivers included for PLCs, DCS, RTUs, Loop Controllers, Bar Code Readers and other serial equipment $\bullet$ Driver or Toolkit available

\section{$6.4 \quad$ Networking}

\section{Iconics}

GEN-NET is an advanced networking protocol designed for the needs of real-time process control systems. It is used to connect GENESIS for Windows and GENESIS Control Series (DOS) workstations. The GEN-NET protocol provides real-time simultaneous network access to process data, alarms, file transfer, and time synchronization.

Reliable Client/Server Design

$$
\begin{gathered}
H N F-1539, \text { Rev. } \\
\text { Paqel6 }
\end{gathered}
$$


GEN-NET uses a distributed client/server architecture that can be easily expanded. This design ensures that the failure of any single node does not affect the operation of others. All data within a GFW node, whether from the RTS, scripting, I/O Server, or DDE, may be accessed by any other node. Graphic displays, reports, data loggers, and trend windows act as clients that automatically locate the data they need from the network.

Simultaneous File Transfer

In addition to real-time data, GEN-NET supports simultaneous file transfer capability. Files can be exchanged between GENESIS nodes while they are running without interfering with the important task of executing other runtime functions. Use this to copy history data to a file server, or import new graphic displays into a system without shutting down.

ICONICS offers a choice of industry standard networks which can be used to implement GEN-NET. Choose ARCNET, NetBIOS or TCP/IP implementations. The NetBIOS version supports all commonly used networks including Ethemet, Novell, Token Ring, DEC-NET, and more. All GENNET functions are available with all network types.

\section{Wonderware}

Wonderware Scout is a client/server Internet tool designed for visualizing industrial automation applications over the Internet and corporate Intranet implementations. Scout extends the highly graphical viewing capabilities found in InTouch and its FactoryFocus enhancement by allowing industrial users to view factory operations at remote sites simply by dialing into the Internet's World Wide Web.

Networking:

Supports any standard NetBIOS network: Ethernet, Novell, Token Ring, Arcnet, etc. DECnet, Serial and TCP/IP connectivity supported.

NetDDE

Using Wonderware's NetDDE, included with InTouch, users benefit from DDE connectivity between applications on different nodes over the network. Connectivity support is provided between Windows, VMS, and UNIX operating environments over a range of network protocols such as NetBIOS, TCP/P, and DecNET, plus serial port communication. NetDDE enables the workstation to be a virtual gateway between network protocols. 


\section{Intellution}

Distributed, Client/Server Architecture -- All FIX products are designed for both stand-alone and networked applications. With our distributed client/server architecture, you can instantly share data across the enterprise, for faster, better-informed business decisions.

View Client

View Clients are ideal for additional operator stations and engineering or configuration workstations. A View Client is networked to one or more SCADA Servers. It has complete read/write access to all data in these SCADA Servers, yet does not maintain a copy of the FIX database.

\section{Citect}

- Supports all NetBIOS compatible networks such as Netware LAN Manager, Windows for Workgroups, Windows NT

-Built-in dual network cable support provides full LAN Redundancy

- Network configuration via simple menu check box

- LAN licensing is based on the number of concurrent users logged into Citect not the number of nodes on the network

- Multi-user system with full communication between operators

-PSTN dial up RAS and WAN fully supported

\subsection{Configuration/Development/Database}

\section{Lconics}

Control and data acquisition functions in GENESIS for Windows are provided by the high performance Real-Time Server (RTS). The RTS scans the V/O, executes the database and control algorithms, checks for alarms, and services all data requests from the GFW applications. Its prioritized, multi-tasking kernel ensures that the most critical functions, such as WO updates, are given the highest priority.

Configuration of the RTS is done using the Strategy Builder. It is an object oriented, icon-driven tool that graphically defines the database functions. The RTS uses advanced function block programming techniques to define all of the VO scanning, calculation, data logging, and control tasks. These function blocks (called algorithms) are placed on the screen and edited with pop-up dialog boxes. Each algorithm has input and output signals that can be used by other blocks in the Strategy simply by drawing connections with the mouse.

$$
\begin{gathered}
\text { HNF }-1539 \text {, Rev.O } \\
\text { Pras } 18
\end{gathered}
$$


Graphical configuration is the fastest way to build sophisticated control and monitoring logic, especially for non-programmers. It's also much easier to maintain and update. If you know how to draw to a flow diagram of your process, you can program it.

The Strategy Builder includes a complete library of over 60 algorithms that provide all of the functionality you need to graphically configure anything from simple MMI systems to sophisticated batch and continuous process applications. The algorithm library is what makes GENESIS for Windows so powerful. Graphical programming is what makes it so easy to use.

All functions, including math, logic, control, and alarming, are graphically programmed using these algorithms. Algorithms are selected from the menu and placed on the screen in the desired size and location.

The GENESIS for Windows Real Time Server (RTS) utilizes a prioritized, preemptive, multi-tasking kernel that insures the integrity of data coming from the plant floor. The RTS always executes its highest priority tasks first, making it so dependable and responsive that you can actually use it to control your manufacturing process from a PC! With more than 250 off-the-shelf interfaces to PLCs, Loop Controllers, and U/O Systems, GENESIS for Windows communicates with more types of plant equipment than any other MMI or SCADA package. The RTS features object oriented, graphical programming that is easy to learn and produces fast results. Configuring a GFW database is as simple as drawing a flow diagram of your process.

The Strategy Builder also features advanced error checking that detects illegal block connections and incomplete databases. Editing tools include grid, gravity, pan, zoom, copy, paste, move, and the ability to convert the Strategy into an AutoCAD DXF file. The copy and paste feature allows portions of existing strategies to be reused. A Strategy can be exported in a dBase format file for editing in other programs such as spreadisheets or databases. The modified dBase file can then be imported back into the GENESIS for Windows system.

At any time during the configuration process, hard copy printouts can be obtained with the click of a mouse. All algorithm definitions and their connections are documented in printed or ASCII file format. Nothing could be easier or faster than graphically programming and documenting your SCADA application with the GENESIS for Windows Strategy Builder!

\section{Wonderware}

Real-time Database tags can be configured as discrete, real and integer values, and strings. There is no limit to the number of each type. Utilities are included for database exporting/importing to/from spreadsheets, other databases, or editors.

$$
\underset{\text { Dace }}{\text { HNF }}-1539 \text { Rev. }
$$




\section{Intellution}

-FIX Draw Workspace -- Build your application quickly and easily from within our single, integrated Workspace environment. In FIX Draw, you can add, modify, delete, and view I/O information. Plus, you can access other system development applications like Historiçal Assign, Display and Recipe. - Graphic Dynamo ${ }^{\mathrm{TM}}$ Wizards -- Create dazzling displays using our library of pre-built Dynamos including pumps, valves face plates, meters, pipes, buttons and check boxes. To use a Dynamo, you simply drag it from the library and drop it into your display. Configuration is simple because Dynamos prompt you for all necessary information. $\bullet$ QuickDraw and QuickView -Enjoy unprecedented speed in your screen development by instantly verifying that your screens work the way you want. On-line Configuration --Build and enhance your application on-line without rebooting or losing any of your valuable data. - Configurable Toolbox -- Customize your toolbox so that the development tools you use most, such as snap-to-grid, align and group, are just a mouse click away. - Templates --Create sizzling screen displays is a snap when you start with our pre-built screen templates. - Command Language -- Create simple or sophisticated sequences of commands using FIX's powerful scripting capabilities.

\section{Citect}

- Extensive Online Multi-media help and Design guide "Fill in the forms" configuration Included Libraries of graphics symbols and templates all user expandable - Express Wizards for communications set-up - Genies for configuration of complex animation objects $\bullet$ Super Genies for configuration and display of repetitive plant processes, that allow common displays to be reused by different sets of variables.

\subsection{Hardware/Software - System Specifications}

\section{Iconics}

Information not available.

\section{Wonderware}

Hardware Specifications:

$\mathrm{PC}$ as required by operating system with a minimum of $8 \mathrm{MB}$ of RAM

Software Specifications:

Windows for Workgroups 3.11 or Windows 95 . 


\section{Intellution}

- Hardware Specifications:

- Computer:

486 or Pentium-based personal computers supported by the manufacturer for Window 95 and Window NT $\bullet$ Memory: 16 megabytes $\bullet$ Disk Space: 200 megabytes $\bullet$ Graphic Support:

VGA, SVGA, or XGA $\bullet$ Mouse:

IBM or Microsoft mouse required for configuration $\bullet$ Networking:

Supports the NETBIOS interface in Windows For Workgroups, Novell and IBM LAN using Ethemet or Token Ring TCP/IP and Serial

- Software Specifications:

- Operating Systems:

Windows 95 and Windows NT v3.51 and greater $\bullet$ Standard Features:

Dynamos,Sample System, Distributed Networking, Object-Oriented Graphics, Historical Trending and Displays, DDE Client and Server,Batch Blocks, and Excel Macros $\bullet$ Options:

Standard Process Control (SPC), Continuous Control, /OO Driver, Recipe Builder, Real-Time SQL/ODBC Interface, Software and I/O Driver Development Toolkits, and More

\section{Citect}

Information not available.

\subsection{Support}

\section{Iconics}

Information not available.

\section{Wonderware}

InSupport provides computer-based assistance for the troubleshooting and repair of complex processes and equipment used in manufacturing. InSupport aids in the diagnosis of equipment and process failures and then presents technical support information to factory technicians on-line, on standard personal computers instead of manuals, books and notebooks. The same multimedia information can be used for interactive training and documentation.

$$
\begin{aligned}
& \text { HNF-1539, RevO } \\
& \text { Page } 21
\end{aligned}
$$


Wonderware's award-winning technical support staff has been rated the best in the industry. Continuing our commitment to customer support, Wonderware offers the Comprehensive Support CD-ROM (CSCD), which expands the concept of support and elevates it to new levels: The CSCD support package includes quarterly software updates, additions to the Wizards library, complete product documentation, sample applications, embedded "how-to" videos and multimedia presentations, news releases, application stories, technical support papers, solutions, and access numbers to Wonderware's Bulletin Board System (BBS).

\section{Intellution}

-QuickStart Tutorial -- With our animated, on-line tutorial, you'll quickly master all the basics of system development as you leam to create customized display screens. $\bullet$ On-line Help -- With a simple click of the mouse, you'll find comprehensive answers to all your automation software questions. Treasure Chest -- Uncover our treasure chest of hot development tips that make building and optimizing your application fast and easy. $\bullet$ Remote Control Software Support.

\section{Citect}

On-Line Support

- Technical Assistance Centre $\bullet$ Training packages $\bullet$ Knowledge Base $\bullet$ Dial-in modem access - Internet Access $\bullet$ Ongoing software maintenance program $\bullet$ Application support $\bullet$ Engineering support

\subsection{Scripting}

\section{Icenics}

All modules feature full Visual Basic for Applications compatible scripting. Or let the Script Wizard write your scripts for you! Based on the world's most popular macro language, Microsoft Visual Basic for Applications, the GENESIS for Windows script language is both powerful and easy to learn. Over 600 commands are available to perform almost any function you can imagine. Use scripts to perform calculations, read and write files, SQL database interface, and much more.

For non-programmers, the ICONICS Script Wizard makes it easy. Hundreds of pre-configured actions are available for selection. Simply fill in the blanks and the Wizard will write the necessary script for you! 
GENESIS for Windows features a powerful scripting language for performing calculations, key macros, or other intelligent functions. Scripting commands let you completely customize how the system responds to process changes and operator actions. The GFW scripting language and commands are based on the world's most popular macro language, Microsoft's Visual Basic for Applications. If you know Visual Basic or VBA, you already know how to write GFW scripts!

Scripts can change screens, generate reports, read and write process data, modify trends, acknowledge alarms, and even play multimedia clips. In addition to over 400 standard VBA compatible commands, there are more than 150 language extensions that support functions specific to the GFW applications. Each script may be defined to execute on a periodic basis, on operator action, time of day, or on an event.

Whether you are an experienced programmer, or just learning, the ICONICS' Script Wizard can help you create useful scripts in minutes. The Script Wizard automatically generates custom scripts based on your menu selections and fill-in-the-blank dialog boxes. With the Script Wizard, you can add powerful custom functions to your SCADA system without any programming knowledge.

The GFW Scripting language supports the concept of global variables. Data in global variables is available to all scripts. This provides a mechanism. for passing data between scripts or for synchronization of scripts. Global variable data can also be incorporated into graphic displays and trends. For example, a graphic display object can be made to rotate based on the value of a global script variable. Or a trend may show the result of a script calculation.

Built-in Editor/Debugger The scripting system includes a full editing environment. The editor enables you to develop and debug your own scripts. Standard windows functions such as cut and paste allow you to take code from one script and move it to another. You can even cut and paste code from Visual BASIC applications.

The Script Editor also includes a dialog box editor and macro recorder. You can easily create custom dialog boxes that prompt the user through a series of steps or selections. Or use the macro recorder to save key strokes and mouse movement for replay.

Scripts can be run and fully tested from within the editor saving considerable debugging time. Debug tools include single step and procedure step. This allows you to execute the script one line at a time. The watch point window displays the value of selected script variables. Break points can be inserted at any point in the code. You have all of the tools you need to follow the actions of a simple script, or a complex one.

A syntax checker points you directly to any problems found in a script before you run it. Syntax is also checked when scripts are saved. All scripts are saved in separate files making it easy to use them in multiple places, or to move them from project to project. 
GEN-USER is the $C$ user task option. GEN-USER provides comprehensive $C$ and assembly language tools that let you implement your own custom programs for execution in the Real-Time Server. Use this option whenever you need to create special functions and programs to complement the real-time control functionality of the RTS. The package includes a variety of functions and libraries supporting access to the real-time database, serial and parallel ports, and file data. A typical user task may include: optimization routines; file format conversion; readiwrite functions with serial and parallel ports; or batch sequences. Use of this option requires a Microsoft $\mathrm{C}$ compiler.

GEN-R\&R is the option for handling custom Reports and Recipe storage within the RTS. This option is supported by the RTS only for compatibility with GENESIS for DOS V3.x strategies. For new systems, it is recommended that GEN-R\&R functions be accomplished using the GENESIS for Windows scripting language.

\section{Wonderware}

Script Editor InTouch provides extensive scripting capability for rapid prototyping, background calculations, and simulation. Condition Scripts include On True, On False, While True, and While False. Button Scripts include On Button Down, While Button Down, and On Button Up. Window Scripts can be invoked when Opened, Closed or While Open. Data Change Scripts activate when data changes value, upon operator actions such as selecting objects, or as the result of events such as alarm conditions.

Scripting is easy. All functions are simply buttons that are adjacent to the script window. All script functions and data variables are chosen by point and click. The script editor itself has Find/Replace and Convert, and it offers up to 256 character expressions for Condition Scripts.

Script functions support logical and mathematical expressions. Users can display single precision floating point numbers, while internal calculations use double precision floating point numbers. New functions have been added such as string manipulation, math functions, file $J O$, system resources, hexadecimal and scientific numerical representations. Users can also write their own script functions and add them to their script menu through the use of the Extensibility Toolkit.

The Recipe option allows users to easily create, modify and download recipes or machine set-up parameters. Process variables such as setpoints, ingredients, process limits, timing, and temperatures, are stored in a.CSV formatted worksheet and can be downloaded to the PLC based upon an event or action. 


\section{Intellution}

Version 6.0 of this batch operating supervisor system offers enhanced recipe management including full version control and tracing and secured development. The planning and scheduling function has been upgraded to provide product plan tracking, created control recipes, and determine batch schedules. Report generation now automatically validated data integrity, security, and online documentation, and batch management offers real-time batch view capabilities, automatic selection of procedures, and pre-start verification.

\section{Citect}

Cicode unleashes the power of Citect. Cicode is a structured language similar to other languages such as Pascal or "C", yet you do not have to be an experienced programmer to use Cicode.

Cicode uses "basic" - like syntax and standard mathematical terminology. This means that Cicode is easy to use. A complete set of mathematical, relational and logical operators are included in the Cicode instruction set.

\section{Welcome to Citect for Windows!}

You can configure a Citect monitoring and control system to suit any industrial application. And because Citect has been designed with flexibility in mind, you can design a system to suit your exact requirements.

Citect suits both small and large applications. Because it is flexible, Citect will keep pace with your plant and information requirements as they change and expand.

yO Optimization

- Operates on a demand basis $\bullet$ Supersedes the conventional predefined scan method $\bullet$ Automatic optimization of block data requests to PLCs and DCs $\bullet$ Automatic rationalization of network wide user data requests $\bullet$ Maximization of control vendors highway bandwidth

\section{Applications Language (Cicode)}

- Fully pre-emptive multi-threaded language $\bullet$ Client Server, Remote Procedure Calls (RPC) $\bullet$ User Written Functions -Indirect Read and Write of tags $\bullet$ Functions are re-usable, modular and transportable throughout the system $\bullet$ Full mathematical and Boolean functions $\bullet$ Full string manipulation If-then-else, For-Do, While-Do, Conditional Executors $\bullet$ Over 500 standard Cicode functions provided $\bullet$ Designed especially for plant monitoring and control applications 
HNF-1539, Rev. O Pase 26 


\subsection{APPENDICES}

Appendix A: $\quad$ Reprint from Control Engineering, February 1997

Appendix B: $\quad$ Reprint from Managing Automation, June 1997

Appendix C: $\quad$ Reprint from Manufacturing Systems, July 1996.

Appendix D: $\quad$ PLCs PLUS Internal Survey

Appendix E: Competitive Review - Paragon TNT

Appendix F: $\quad$ Reprint from InTech, May 1996

Appendix G: $\quad$ Reprint from Design Engineering, June 1996

Appendix H: Reprint from Measurements and Control, April 1996

Appendix I: $\quad$ Reprint from Computerworld, May 1996

Appendix J: $\quad$ Reprint from Maintenance Technology, May 1996 


\begin{tabular}{|c|c|c|c|c|c|c|c|c|c|c|c|c|c|c|c|c|c|c|c|}
\hline \multicolumn{2}{|c|}{$\begin{array}{l}\text { CONGROL } \\
\text { ENGNEERING } \\
\end{array}$} & \multicolumn{7}{|c|}{ Type } & \multicolumn{4}{|c|}{$\begin{array}{c}\text { Operating } \\
\text { System }\end{array}$} & \multicolumn{6}{|c|}{ Features } & \\
\hline $\begin{array}{l}\text { Whe } \\
\text { Process Manag }\end{array}$ & ement Software & \multirow[b]{2}{*}{ 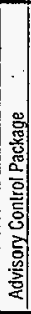 } & \multirow{2}{*}{\multicolumn{2}{|c|}{ 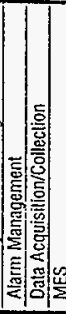 }} & \multirow{2}{*}{\multicolumn{3}{|c|}{ 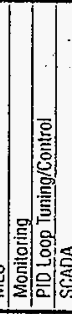 }} & \multirow[b]{2}{*}{ tion } & \multirow{2}{*}{\multicolumn{4}{|c|}{ 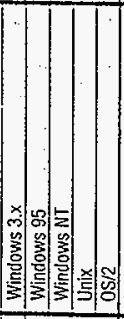 }} & \multirow{2}{*}{\multicolumn{2}{|c|}{ 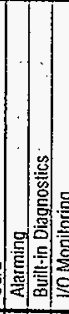 }} & \multirow{2}{*}{ 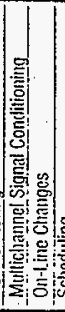 } & \multirow{2}{*}{\multicolumn{2}{|c|}{ 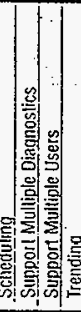 }} & & \\
\hline Company & Product & & & & & & & & & & & & & & & & & & \\
\hline $\begin{array}{l}\text { 8V Technologies, Inc. } \\
\text { (Brook Pk., O.) }\end{array}$ & $\begin{array}{l}\text { BVC ToolSet for } \\
\text { Rapid Application Development }\end{array}$ & & $x$ & & $x$ & & & $x$ & $x$ & & $x \mid$ & & $x \mid x$ & & $x|x|$ & $|x|$ & $|x|$ & $x$ & 427 \\
\hline 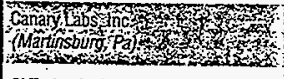 & frend 1 now & & $\approx$ & & $\ddot{x}$ & & & & $\bar{x}$ & $x$ & $x$ & & 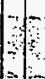 & 1 & 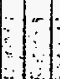 & 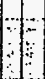 & & $x$ & 428 \\
\hline $\begin{array}{l}\text { Ci Technologies, Inc. } \\
\text { (Freeport, N.Y.) }\end{array}$ & Citect . & & $x$ & & $x$ & & $x$ & & $x$ & $x$ & $x$ & & $x$ & $x$ & $x$ & & $x$ & $x$ & 429 \\
\hline 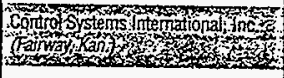 & Ucos Distributed control System & $\because$ & $x$ & & $x$ & & $x$ & & & 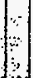 & $|x|$ & & $\underline{x}$ & & & & $x$ & $x$ & $\begin{array}{r}430 \\
46\end{array}$ \\
\hline $\begin{array}{l}\text { ControlSott, Inc. } \\
\text { (Cleveland, 0.) }\end{array}$ & $\begin{array}{l}\text { INTUNE } \\
\text { Mantra } \\
\text { QuickTune }\end{array}$ & & & & & $\begin{array}{l}x \\
x\end{array}$ & & & $x$ & & $x$ & & $\left.x\right|^{x}$ & & $x\left|\begin{array}{l}x \\
x\end{array}\right|$ & $x x^{x}$ & $x \mid x$ & $\begin{array}{l}x \\
x\end{array}$ & 431 \\
\hline 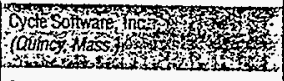 & 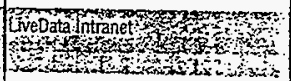 & & $x$ & & & & & & $\therefore$ & $x$ & $x$ & x. & $x$ & & $\left(\begin{array}{l}x \\
x \\
7\end{array}\right.$ & & $x$ & $x$ & 432 \\
\hline $\begin{array}{l}\text { DataMyte/Rockwell Automation } \\
\text { (Minnetonka, Minn.) }\end{array}$ & Quantum SPC Software & & & & & & & $x$ & $x$ & $x$ & $x$ & & $x$ & & & & & $x$ & 433 \\
\hline 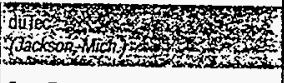 & 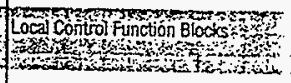 & $\%$ & & & $x$ & $x$ & 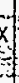 & & $x$ & $\prod_{1}^{x}$ & $x$ & 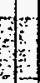 & $x$ & $|x|$ & $x$ & & & 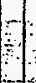 & 434 \\
\hline $\begin{array}{l}\text { ExperTune inc. } \\
\text { (Hubertus, Wis.) }\end{array}$ & $\begin{array}{l}\text { Multi-Channel Loop Analysis } \\
\text { and PIO Tuning } \\
\text { PID Loop Analyzer and Tuner }\end{array}$ & & & & & $x$ & & & $\begin{array}{l}x \\
x\end{array}$ & $x$ & $x$ & & $x$ & $x \mid$ & $x|x| x$ & & $|x|$ & $x$ & 435 \\
\hline 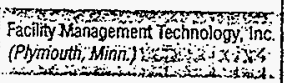 & 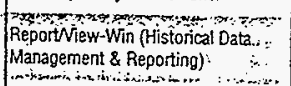 & $x$ & & & $x$ & & & & $x$ & $x$ & 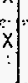 & & $\cdots$ & $x$ & & & $\mid$ & $x$ & 436 \\
\hline $\begin{array}{l}\text { isTech Integration, Inc. } \\
\text { incoln, Mass.) }\end{array}$ & SPCworks & & & & & & & $x$ & & & $x$ & $x$ & $x \mid$ & & $x$ & $x$ & & $x$ & 437 \\
\hline 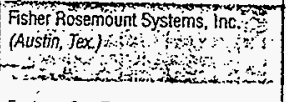 & $\begin{array}{l}\text { Provox Process Automation System } \\
\text { Delta V (scalesabi Process Nutomation System) } \\
\text { RS3 Process Automation System }\end{array}$ & $\begin{array}{l}x \\
x \\
y\end{array}$ & $\begin{array}{l}x \\
x\end{array}$ & & $\begin{array}{l}x \\
x \\
x\end{array}$ & & & & $\begin{array}{l}x \\
x \\
x\end{array}$ & $x$ & $\begin{array}{l}x \\
x \\
x \\
x\end{array}$ & $x|x|$ & \begin{tabular}{l|l}
$x$ & $x$ \\
$x$ & $x$ \\
$x$ & $x$
\end{tabular} & & \begin{tabular}{l|l|}
$x$ & $x$ \\
$x$ & $x$ \\
$x$ & $x$
\end{tabular} & & $\mid \begin{array}{l}x \\
x \\
x\end{array}$ & $\begin{array}{l}x \\
x \\
x \\
x\end{array}$ & 438 \\
\hline $\begin{array}{l}\text { Foxboro Co., The } \\
\text { (Foxboro. Mass.) }\end{array}$ & 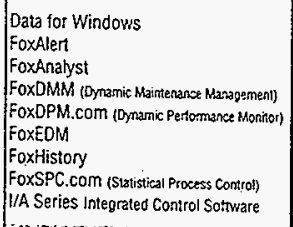 & $x$ & & & $\mid \begin{array}{l}x \\
x \\
x\end{array}$ & & & & & $\mid \begin{array}{l}x \\
x \\
x \\
x \\
x\end{array}$ & \begin{tabular}{l|l}
$x$ & $x$ \\
$x$ & $x$ \\
$x$ & $x$ \\
$x$ & $x$ \\
$x$ & $x$ \\
$x$ & $x$ \\
$x$ & $x$ \\
$x$ & $x$
\end{tabular} & $\begin{array}{l}x \\
x \\
x \\
x \\
x \\
x \\
x \\
x \\
x\end{array}$ & \begin{tabular}{l|l}
$x$ & $x$ \\
$x$ & \\
$x$ & $x$ \\
$x$ & $x$ \\
$x$ & \\
$x$ & $x$ \\
$x$ & $x$
\end{tabular} & $x$ & $\begin{array}{l}x \\
x \\
x \\
x \\
x\end{array}$ & $x \mid x$ & $\mid \begin{array}{l}x \\
x \\
x \\
x \\
x \\
x \\
x\end{array}$ & $\mid \begin{array}{l}x \\
x \\
x\end{array}$ & 439 \\
\hline $\begin{array}{l}\text { GE Fánic Automation } \\
\text { (Chatotlesville, Va) } \\
\text { and }\end{array}$ & CIMPLCTY HMM & & 18 & & $x$ & & & & & $x$ & $x \mid>$ & & & $x$ & $x$ & & $x$ & $x$ & 440 \\
\hline $\begin{array}{l}\text { Gensym Corp. } \\
\text { (Cambridge. Mass.) }\end{array}$ & GDA (G2 Diagnostic Assistant) & & $x$ & & $x$ & & 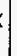 & & & & $x \mid>$ & $x$ & $x$ & $x$ & $x \mid$ & & $x$ & $x$ & 441 \\
\hline
\end{tabular}




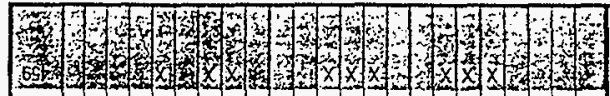
$\int x|x| x|x| x|x \quad x| x|x| x|x| x|x| x|x| x \mid x$

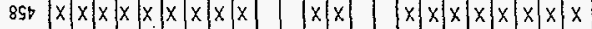
3.

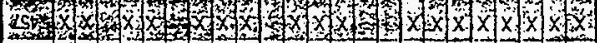
9St

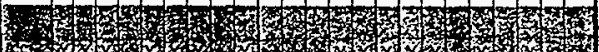

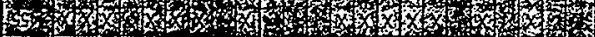

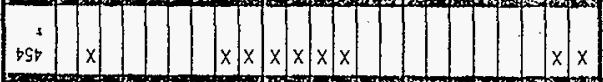

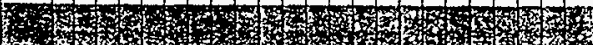

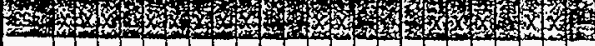
\begin{tabular}{l|l}
$x$ & $x$ \\
$x$ & $x$ \\
$x$ & $x$
\end{tabular} 25\% $x \mid x$ $x \times x \mid \begin{aligned} & x \\ & x \\ & x\end{aligned}$ $x \mid x$

8.9.5.

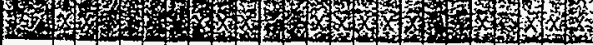
ost $x|x| x|x| x \int x x_{x} \cdot x$ (x) (4) $8 \sin x \times x|x| x|x| x|x| x|x| x$

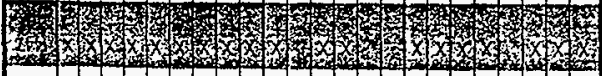
$960 \quad x\left|\begin{array}{l}x \\ x\end{array} \quad x \leq x\right| x \mid x$

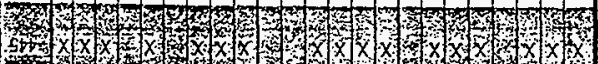$$
\text { st }
$$$$
x: x \times x \times x|x| x|x| x \mid x
$$

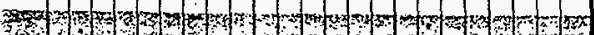

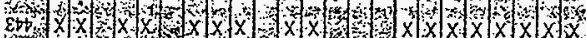
$200 x|x| x|x| x|x| x|x| x|x| x|x| x|x| x$

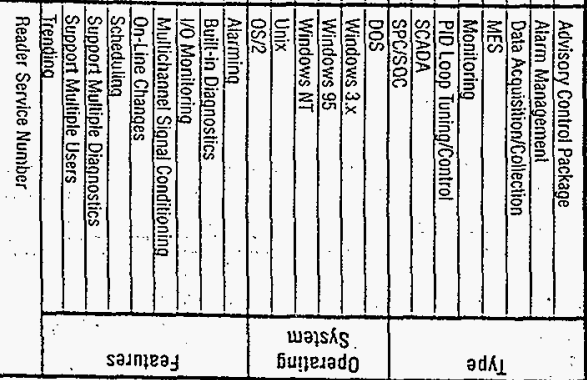

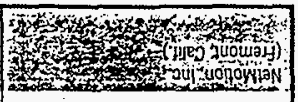

jnoxpo7

MainวBpบg

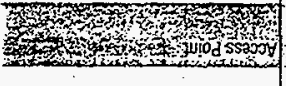

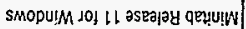
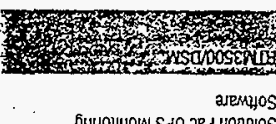

EuบoluOW Son aed uonnos

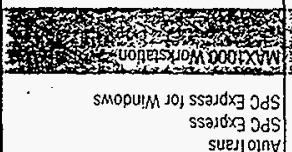

SURJiolny

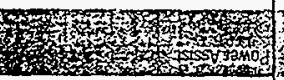

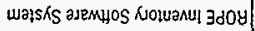

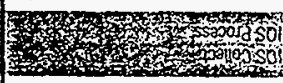

$X I 5$

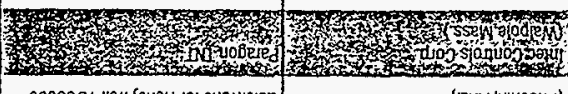

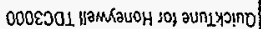

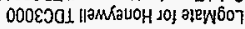

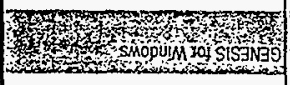

0008 บеग

(ZIJV'Xivaoud)

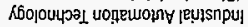

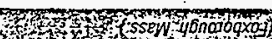

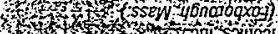

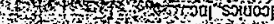

(2!) 'X!)

วท] II MauoH

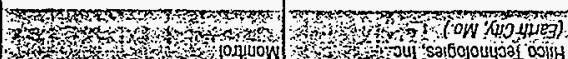

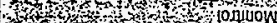

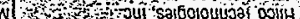

( PW 'SIIIW \$6U!MO)

swass/s voṇen/ogul

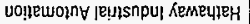

fonpodd

Aueduor

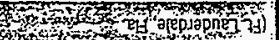

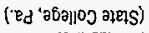

(' YSEM '/lay)og)

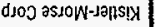

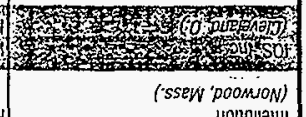

(IIIPJ 'ESan bisoj)

("yo!w 'platyunos)

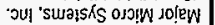




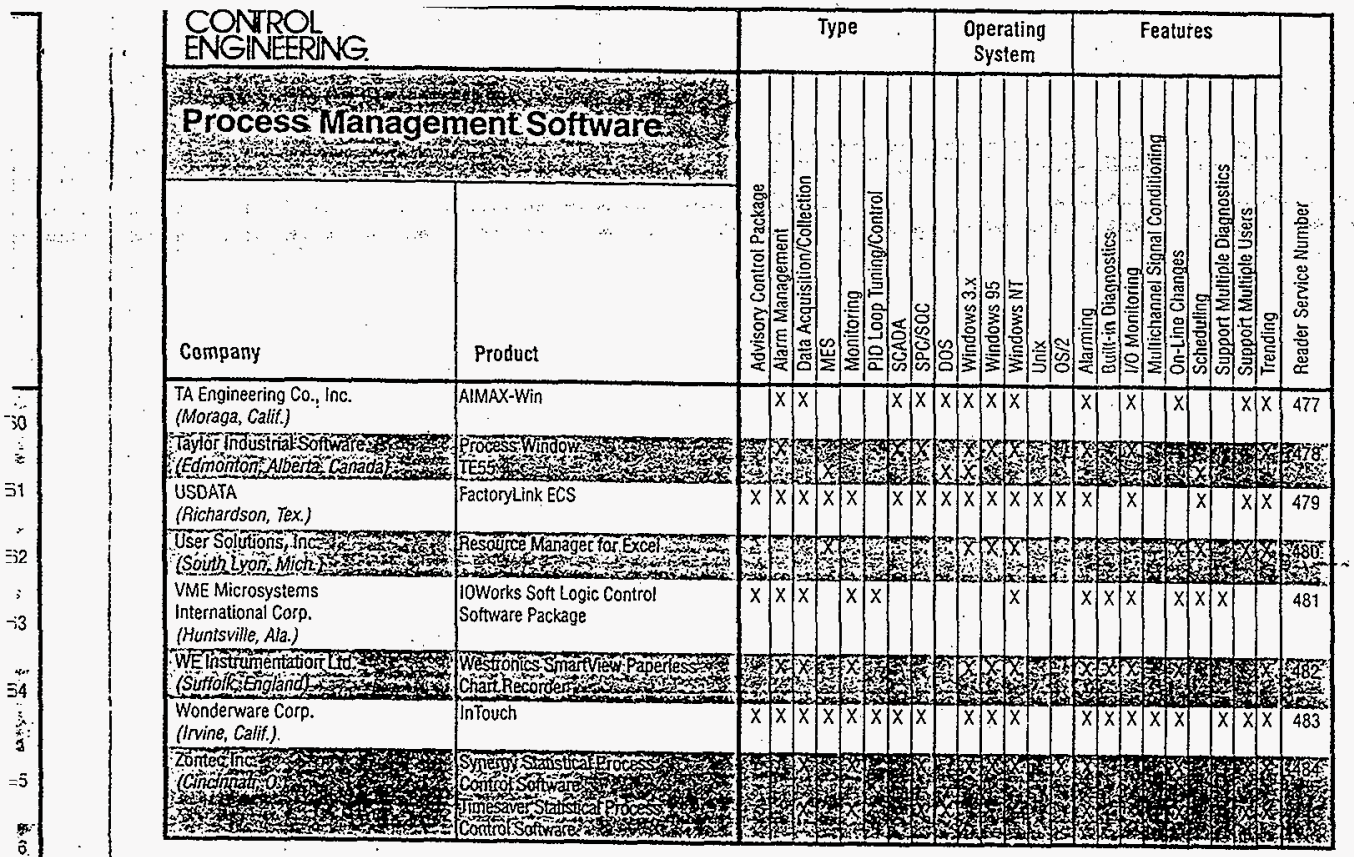

TA Engineering $\mathrm{Co}_{0 .}$ Inc.

(Moraga, Calif.)

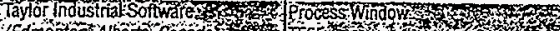
(Edmonton Albeta Canadá USDATA

(Richardson, Tex.)

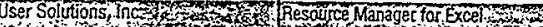

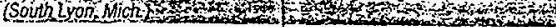
VME Microsystems international Corp. 10Works Soft Logic Control

(Huntsville, Ala.) S Su Wonderware Corp. (Irvine, Calif.).

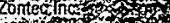

(cincmatom 03

$$
\begin{aligned}
& \text { ANF-1539, Rev.O } \\
& \text { Page } 30
\end{aligned}
$$


range of factory-floor products designed to link manufacturing operations to business management Process Window is a graphical MMI system while TESS is a constraint-based finite scheduling tool operating on real-time updates from the shop floor. ...Ro* 1s4

\section{TETRAWTERMTIOMAL.}

Location: Houston, TX Product Chameleon 2000, CS/3. Ap plications: ERP. Outlook: Tetra has an opportunity to capitalize on the growth in mid-range, client/server ERP systems. It has focused on electronics and CPG companies with large numbers of international sites. Iike some others, it is hoping these companies will want to standardize on a single package that can quickly be deployed in distributed sites. BC\# 195

\section{TSWITERTATONAL}

Lacation: Atlanta, GA. Product Enterprise MPAC. Applications: CMMS. Outlook Enterprise MPAC defines a new approach to asset care with a robust client/server system designed to link users across the enterprise with financial, personnel, asset, and inventory data Open architecture links EMPAC with third-party applications from Oracle, SAP, and PeopleSoft. TSW' Web strategy, TSWNet, adds Java applets for RFQs, supply chain management, and electronic document retrieval. Re: 18

\section{USOATA}

Location: Richardson, TX. Product: FactoryLink ECS. Applications: MMI, SCADA, MES. Outfook: USDATA has de
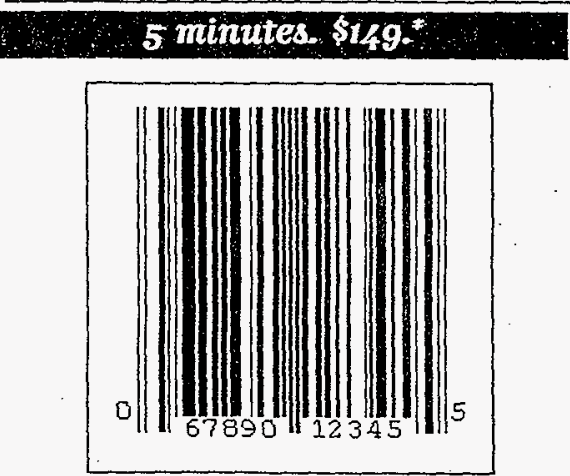

This label is correct.

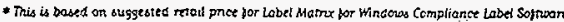

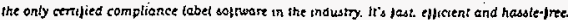
And that's a guarante.

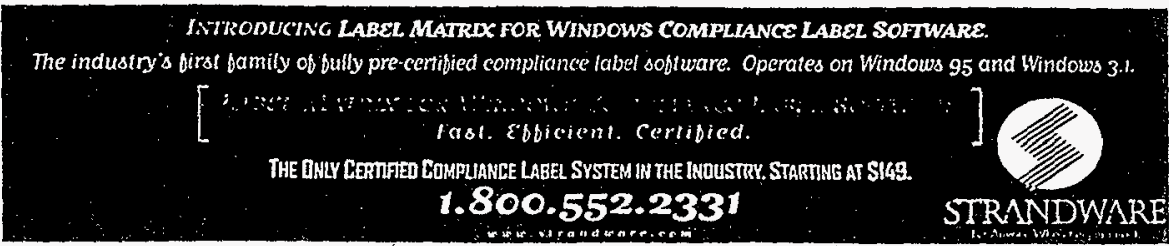

READER CARD NUMBER 48

B-1

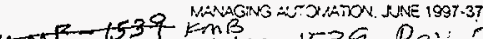

veloped an open, scalable, and modular software system for building real-time industrial applications in Microsoft Windows NT. FactoryLink ECS can be used to create easy-to-use, highspeed, graphical applications from a single process to an enterprise-wide, multinational operation: Major features of the system include graphical monitoring and control; enterprise database connectivity, and a real-time engine for core functionality such as timing and counting.

B.. 197

\section{VISIBILTY WA.}

Location: Wilmington, MA. Product: Visibility. Applications: ERP. Outlook: Visibility offers a powerful, graphically rich ERP program directed specifically at make-to-order and engineer-to-order environments. The Visibility software package forms a completely integrated suite which includes financial, material management, project management, cost estimating, and field service applications for customer-driven manufacturers. BC\# 198

\section{WONDERWARE CORP}

Location: Irvine, CA Product: InTouch, InTrack Applicacomplete suite of software products for industrial and process automation under Microsoft Windows NT. InTouch is a true 32bit, object-oriented graphical interface. In Track is a full-featured production management system for tracking work in process. These and other Wonderware products can be integrated into a single, tightiy bundled applications suite with Factorysuite development tools.........................................................
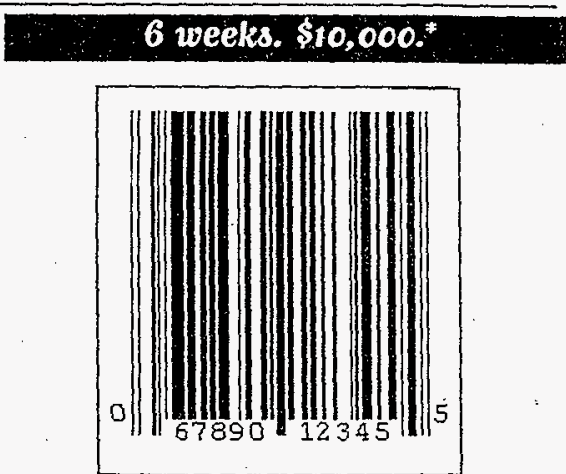

This label isn't.

* This is based on 6 weets of resubmistons and red tope elong with a slo.000 fine for compllance bar cade velation. Doing comptiance tobels the old way just doesn't pay. Even th you de set it nght it's not last not etficient and not hoste-tree. tions: MES, MMI, SCADA. Outlook: Wonderware offers a 
control and access to multiple inventory locations and individu. alized reordering of equipment, parts, and supplies. ...ACH 117

Location: New Providence, NJ. Product MLMI Applications: Finite capacity scheduling. Outlook: Chesapeake has probably done more than any single software supplier in the process industries to analyze the dynamies of the supply chain and its effects on manufacturing and distribution of chemicals, oil and gas, and formulated products. Chesapeake has a highly experienced im. plementation team and strong partnerships. REA 111

\section{BTEAHOLOAIES}

Location: Fairport, NY. Product CITECT. Applications: MES, finite capacity scheduling. Outlook: CITECT is a Windows-based MMI for discrete and repetitive manufacturing. Version 4.0 makes it possible to build a high-speed automation network using $P C$ s running any flavor of Windows, and can accommodate up to 40,000-point SCADA systems. IIC: 112

\section{BITISION}

Location: Torrance, CA Product CIM+. Applications: MES, SPC, warehouse management. Outlook: CM vision offers a unique combination of integrated plant-floor and warehouse so lutions that collect, monitor, and control data flow. CIM+ supports a distributed client/server architecture developed with an "open" toolset supporting multiple databases and platforms. RCH 113

\section{CHROM SYSTEMS ING}

Location: Cincinnati, OH. Product Control, ControlAcquire Applications: ERP. Outlook After nearly 30 years as a software supplier, Cincom has staked out the highly engineered product market. ControliAcquire is designed to marage the entire sales cycle, including prospecting, product configuration, cost estimating, and quoting, and integrate it within the enterprise............RC 114

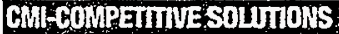

Location: Grand Rapids, MI. Product TRANS4M. Applications: ERP. Outbook: Few ERP software vendors concentrate as completely on one manufacturing environment as CMI does on repetitive manufacturing. TRANSAM is a thoroughly integrated system for the needs of manufacturers in such industries as plas tic injection molding, rubber and plastic extrusion, sand and die casting, metal stamping, and assembly. ..RE 115

\section{BOMPUIER TEBHMOLOGY BORP.}

Location: Milford, OH. Product: Interact. Application: MMI, SCADA Outlook: CTC offers one of the easiest-to-use MMI systems available, on both PCs and its own touch-screen workstations. Interact software modules are open and flexible enough to rin in any combination on CTC's PowerStations...........RE 116

\section{BATSTEEMSTSEISWR}

Location: Greenville, SC. Product: MP2 Enterprise. Applications: CMMS. Outtook: Datastream takes CMMS to enterprise level with a 32-bit, Windows NT-based, client/server asset management system which scales up easily and runs across intranets. version 5.0 supports global inventory management, allowing

\section{DATAWORTS BOAP:}

Location: San Diego; CA Products: ManFact II, DataFlo, Vantage, Vista, Enterprise Server. Applications: ERP, MRP II. Outlook: DataWorks has an unusual breadth of ERP products, from Vista, an easy-to-use Windows-based system, to Enterprise Server, a sophisticated solution offering distributed client/server computing based on object technology. Vantage is for the order-intensive mid-range manufacturer with a mix of custom and standard part orders and multilevel assemblies. ManFact $\Pi$ is designed for larger job shops and make-to-order organizations. DataFlo contains 32 sophisticated modules for deep ERP solution in high-volume, repetitive, and discrete manufacturing...... BCH 118

\section{DEMAND MAMAGENENT ING.}

Location: St. Louis, MO. Product: Demand Solutions. Applications: Demand forecasting and planning. Outlook: Operating primarily in the make-to-stock as well as assemble-to-order and manufacturing distribution environments, Demand Management offers a highly functional product based on Windows NI and 95. It is capable of multisite management and consolidation, and offers workflow-enabled routing of documentation. ...........RC 119

\section{DP SOLIIONSTW.}

Location: Greensboro, NC. Product: PMC for Windows. Applications: CMMS. Outlook: DPSI has one of the most complete CMMS solutions, using client/server technology and written in Microsoft Access. Through the Frank J. Meitz Maintenance Management Institute, DPSI does exceptional training and evangelism work for advanced CMMS, emphasizing the importance of maintenance management in the enterprise.........................RC\#120

\section{EFFE TIUE MANAGEMETI SYSTEMS}

Location: Milwaukee, WL Product Time-Critical Manufacturing. Applications: ERP, MRP II, MES. Outlook: EMS combines enterprise planning and shop- floor control in one "preintegrated" product suite with machine tool interfaces, SPC, and engineering interfaces. EMS believes this unique strategy saves significant time in achieving retums and benefits from the software by elim. inating expensive enterprise-wide system integration.......... 121

Location: Kanata, Ontario, Canada Product: webPLAN. Applications: Advanced planning and scheduling. Outlook: No other company is trying to establish a manufacturing software product in the market by giving it away over the internet. The basic webPLAN product is free, and a number of modules are available for materials planning, consolidating procurement across multiple sites, etc. EPS is firmly entrenched in real-time planning over the Web. RC: 122

\section{EXPANDABLE SOFTUAR}

Location: Santa Clara, CA. Product: Expandable Sofware. Ap. plications: ERP. Outlook: Expandable Software is extremely strong in global, repetitive manufacturing and offers one of the

\section{EITERPRISE PLAMNIN STSTEMS TIT.}


further by the recently released Constructor. Agile planning is also possible with a feature allowing concurrent co-product planning, which calculates the optimum production of co-prodLocation: Hyannis, MA Product: Process Enterprise Resource/Planning (PERP). Applications: ERP. Outlook: Formerly Software 2000, Infinium is capitalizing on the growth in ERP for process manufacturing. Infinium offers a full range of client/server financial; HR/payroll, materials management, and process manufacturing applications. As a result, the company enjoys a $90 \%$ plus customer retention rate.

RG: 135

\section{IWTELIUTION ING.}

Location: Norwood, MA Product FIX BOS. Applications: MES, MMI, SCADA Outlook: Intellution's FIX family of products for MMI and SCADA applications is considered one of the best engineered on the market. Intellution was one of the first out of the gate with a 32-bit Windows NT product and claims to have more 32-bit installations than all other automation software vendors combined. FDX BOS is the first PC-based automation solution to seamlessly integrate a batch manufacturer's SCADA operations with higher-level MES functions. fint 138

\section{DIEMTA NORTHAMERIGA}

Location: Rosemont, IC. Product: Movex Applications: ERP. Outlook: Intentia offers a fully integrated business application sys tem covering logistics, manufacturing, finance, product development, marketing, and human resources. Movex operates in make-to-stock, assembletoorder, configure-to-order, engineer-toorder, repetitive, make-to-demand, batch process, continuous process, and manufacturing distribution. The company is devoted exclusively to the $\mathrm{AS} / 400$ platform................................. 137

\section{IITERAGTIVE GROUP}

Location: Burlington, MA Product JIT Enterprise, InfoFlo. Applications: ERP. Outlook: Concentrating on to-order manufacturing, Interactive offers two complementary products. InfoFlo incorporates a rules-based product configurator for estimating, quoting, engineering, and order processing of highly engineered products, with integrated financials in a client/server environment. JT Enterprise Systern is a native Oracle-based solution for discrete manufacturers with special features for contract manufacturing such as aerospace and defense. RC\# 138

\section{MUUTUE MANIFAGTURWG:}

Location: Kirkland, WA Product: MRP9000. Applications: ERP, MRP II, shop floor execution. Outlook: A spin-off from PRO:MAN, one of the earliest providers of UNDX-based MRP II software, Intuitive offers a completely integrated ERP/MRP solution especially suitable for small and medium-sized manufacturers. Totally Windows- and Windows NT-based, it combines MRP II functionalities with a strong emphasis on ISO 9000 quat ity-assurance features. RC* 139

\section{JBA WTERMATOKAL}

Location: Rolling Meadows, IL Product: System 21 Manufacturing. Applications: ERP. Outlook JBA has founded its fut Iy integrated ERP suite on JIT, TQM, and continuous-improvement techniques. It is completely scalable and adaptable with open, client/server, and object-oriented technologies, enhanced ... RG: 140

\section{JGIT}

Location: Englewood, CO. Product: Demand Flow. Applications: MRP, MRP II. Outlook: The John Costanza Institute of Technology is devoted solely to the implementation and advancement of non-scheduling-based manufacturing. Its flow manufacturing techniques are used around the world to speed product manufacturing and delivery. Emphasizing kanbans, group technology, customer responsiveness, and total employee involvement, the systerm comes with hardware, software, and training included. RC. 141

\section{DB. ENWARDS:CO}

Location: Denver, CO. Product: OneWorld, ERPX. Applications: ERP. Outlook: One of the fastest-growing companies in ERP, J.D. Edwards has made extensive forays into process manufacturing industries. It is leading in adoption of client/server with a migration path for users called Configurable Network Computing, an architectural foundation designed to insulate users from the technical details of client/server. OneWorld also has strong mixed-mode manufacturing capabilities. RCH 142

\section{JMLWDUSTRIES}

Location: Robesonia, PA. Product: Front Office. Applications: ERP: Outlook: JNL has one of the most complete manufacturing management systems avajlable, with a unique, Windows-based product packaging information for the shop floor and communicating it to the proper people on the plant floor. Front Office has workflow, demand replenishment, pricing and promotion, and multicurrency capabilities..........................R* 743

\section{JOBBOSS SOFWMARE}

Location: Minneapolis, MN. Product JobBOSS for Windows. Applications: MES. Outlook: JobBOSS offers a very comprehensive business management and shop-foor control system for make-to-order and contract manufacturers. The Windows-based software handles all aspects of a job-shop environment, with both 16- and 32-bit processing. Offers full Internet support.... Rc* 144

\section{Giomios IIT.}

Location: Waitham, MA Product: ShopTrac, Timekeeper/AS. Applications: MES, shop-floor control. Outlook: Kronos offered the first computerized timekeeping and attendance system in the 1980s, with ShopTrac. now a Windows product. The Timekeeper/AS Labor Data Collection module is developed exclusively for the IBM AS/400 to measure labor content of every job in-house, track work in process, allocate labor, and maintain quality control through feedback on scrap and rework.......Rc 145

\section{LAMNER GROUP}

Location: Cleveland, OH. Product: Provisa Applications: MES, fnite-capacity scheduling. simulation. Outiook: Formerly AT\&T Istel, Lanner's finite capacity software has the advantage of

$$
\begin{aligned}
& 14 \text { NF-1539, MaNaGiNg AUTOMATON, JUNE 1997.27 } \\
& \text { Roge } 33
\end{aligned}
$$




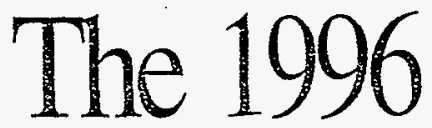

Manufacturing

Systems

Conmunicating computers, intra-office and worldwide

Kevin Parker

Edito:

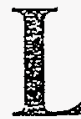

OKNG AT THF TOP SOI STMC FOR 1995

one is struck by the teslization that the ite business of the top softhere sysiem rendors

is communications. What stared ous as $2 n$

inveniory-lacking tool has become enter.

prise resources planning (ERP). Maintenance management susiems are fully integrcied with financial and procuciton razagerent systems. Superisory control is distributed process menagement. Doeument managencat systems are a communicotions shibstrale. Supp) y-chein sofurare shares information tha: wed to be hos ded. And so it goes.

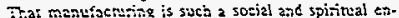
ceavor is what makes it so fascinzting. Yes. things get $m \geq 0=$ through the execution of various physicel processes. But none of thet could ceise place if is vieren" $i$ for the aecumilation and communication of information, all different kinds of information.

Looking at the numbers

The maior markets for manufacliting softurate systems. onee CAD has been elimirated from corsideration. a: $\equiv R P$. supervison control. maintenance management, ware house management. and supply-chain soituare. Like 3ry list thai siars with a compeny that has nonutactiring software-reiated aventes of 51.3 bilfon act ends

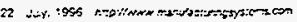
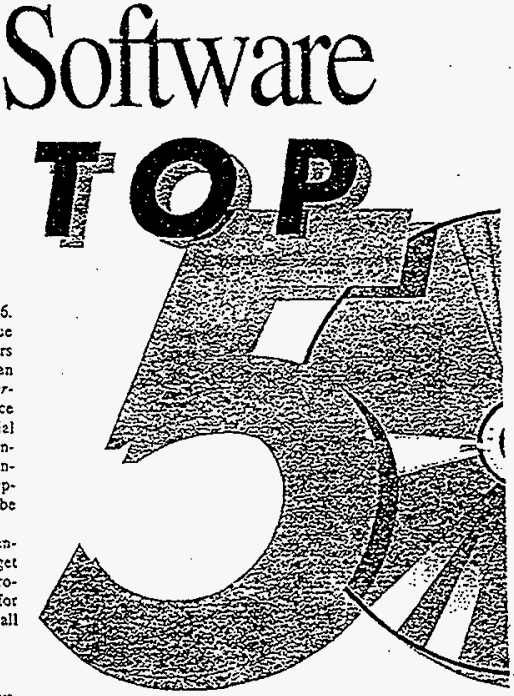

with a company iaking in a respeciajle 519 million. it covers sonsiderabic greind.

The Top 50 in 1994 ineided compatics with soltwere. related evenues of nezi:y $\$ 3$ billion. In 1995, the lisied revenues inceased to S5.= billion. Fis yea: the number 
is $\mathrm{S} 5.6$ billion. A remarkable increase, bearing in mind that the very large CAD vendors have been removed from the list.

Besides the Top 50, the magazine in this issue profiles 10 additional software suppliers. Of the total 60 companies, 36 are ERP vendors. The market for ERP systems is huge, and expanding. It's been said that there are as many as 250 ERP/MRP II software vendors worldwide. Market analysis speculate as to when an eventual consolication will occur, but as new software is developed the suppliers continue to successfully differentiate themselves from one another. Eventually, this ability to specialize will result in a market that consists of many vendors that provide a specific functionality, say, order entry for consumer packaged goods, and a smaller group of "framework" providers that put together solutions consisting of basic functionalicy and the appropriate applets.

If you include "intelligent real-time systems" provider, Gensym, five companies profiled in the Top 50 are primarily in the business of supervisory control. Having begun as PC-based operator interfaces, supervisory-control systems have grown to take on a range of processmanagement, and even production-management, capabilities. The largest vendors, Wonderware, Rockwell Software, Intellution, and USDATA have lately taken to bundling suites of products that include the

bundling suites of products that include the
operator interface, supervisory control,
batch control, and what's now being
called "Soft PIC," in other words,
PC-based control.
so incluce PSDI. TSW International, Datastream Sysiems, and Revere.

Warehouse-management systems also have become more sopristicated. able to handle push. puil, or hybrid environments, and with specific functionaliiy appropri- ate to a wide range of industries and operztional modes. Warehouse managenent companies profiled inclice $\mathrm{HK}$ Systems, Mchugh Freeman. Catalyst International, and Optum Software (formerly American Tunkey). It should be noted that several ERP and supply-chain vendors are also known for their warehousing systems, including Computer Associates, Marcam, JBA, J.D. Edwards, SAP, IMI, and Manugistics.

The other supply-chain companies are a bit of a mixed bag, although one of the most interesting mixed bags you could ever hope to find. The supply-chain section includes Manugistics, IMI Norh America, Numetrix, and i2 Technologies. IMI's product, System ESS, is a cusromer-oriented transactional systern, while Manugistics and Numetrix have primarily decision-support applica-. tions that use memory-based processing to do forecasting, demand planning, distribution management, plant production scheduling and planning, transportation management, and supply-chain optimization. i2 Technologies is a planning and scheculing vendor that has introduced a system for optimizing supply chains, and even multiple supply chains.

Last, but not least, three companies, SAS Institure, FileNet, and Cimlinc, are primarily concemed with document management, though their approaches vary widely.

\section{What follows}

As noted, there are actually 60 profiles inciuded in the Top 50 profile section. A list of the additional compenies appears in a supplemental listing on page 131 . In addition to that, a sofiware directory of many other of the top software suppliers surveyed for the Top 50 appears on page 133.

Ranking of the Top 50 is based on revenue derived from manufacturing sofiware in the calendar year 1995. This includes revenues associated with taining, services, and support, eamed anywhere in the woild. Companies wishing to compete in the Top 50 completed questionnzires that asked for 1995 total revenues, as well as revenues related to manufacturing software applications oniy. Sorne vendors could not complete the form, either because their companies are privately held or for other reasons, and have been left off the list.

The survey was conducied by Plant-Wide Research Corp., North Billerica, Mass. The task was a formidable one, calling for both organizational skills and knowiedge of the manufacturing software markets.

It has proven both interesting and enligintening to watch the changes in manufacturing software companies over the last several years. The Top 50 serves as an indication of where this ver important industry is iodzy, and where it wijl be going iomorrow. More importanily, the listing can serve as a cool to help managers make informed decisions involving product technologies and vendors. The company p-oniles are snapshots of societal effortsat the same time both cooperative and competitive-to achieve a revolution in information technology. 
Web browers, or through company

Intranets. With WebMiner, users can pull data from virtually any source on the World Wide Web and have WebMiner use its intelligence to make suggestions about how that data would be useful to the operation of the business.

While most of its software is designed to improve production processes, Gensym recently released a product with a slightly different goal: the modeling and optimization of business processes. Moore says this product, called ReThink, "takes the ability to do object modeling and extends it to the entire supply chain."

He says Rethink users can put business processes into an object-based model-just as $G 2$ users do with production processes-and let the system work out the best method of arranging those processes to improve a company's efficiency.

"Our process-level models estimate states that can be measured, such as quality or emissions levels," Moore explains. "We have taken that rechnology up to the enterprise level. There, we can treat supply chains like processes by estimating how various behaviors within an organization affect productivity," Moore says.

ReThink is a perfect iool for companies considering business process reengineering. Xerox Corp. used ReThink to help streariline its purchasing process, and Moore says IBM and EDS have expressed interest in having their consulting groups use it to aid their clienis' reengineering efforts.

The system lets groups of managers collaborate-even from different locations-to build graphical models of a business process, then run simulations to see how the process performs under real-world conditions. "They can test different scenarios unilil they find the one works," Moore says. "Then they can use it as an ongoing model to monitor the pro. cess, and to diagnose problems when necessary."
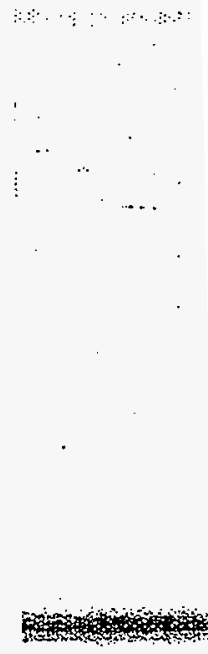


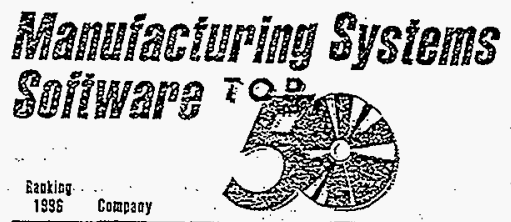

\begin{tabular}{|c|c|c|c|c|}
\hline 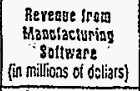 & $\begin{array}{c}\text { Percept Revenas } \\
\text { incm Mastiocturlag } \\
\text { Solthare }\end{array}$ & 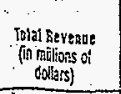 & 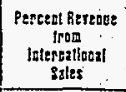 & Rsabiab \\
\hline 1,320 & $4 \%, 70$ & 40,367 & 80 & 31 \\
\hline 735 & $23 \%$ & 3,200 & $50 \%$ & 2 \\
\hline 56 & Sistos & 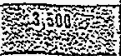 & $x^{2} 5 \%$ & 6y \\
\hline 394 & $100 \%$ & 394 & $68 \%$ & 3 \\
\hline 25 & Kan & 545 & $2,32 \%$ & $\gamma / 13$ \\
\hline 216 & $100 \%$ & 216 & $70 \%$ & 12. \\
\hline 203 & 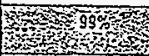 & 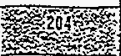 & 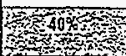 & $\sqrt{3} 9$ \\
\hline 180.6 & $25 \%$ & 562 & $52 \%$ & 14 \\
\hline$\frac{15}{134}$ & 4,1096 & 134 & 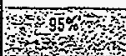 & 67 \\
\hline 130 & $60 \%$ & 215.5 & $34 \%$ & $t$ \\
\hline 124 & 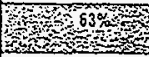 & 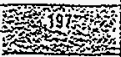 & (a) & $16,16, y$ \\
\hline 76 & $32 \%$ & 240 & $48 \%$ & 21 \\
\hline 3. & S 67 & 3514 & ores & (7) \\
\hline 62 & $75 \%$ & 83 & $13 \%$ & 18 \\
\hline$x$ & 370 & 3. & Paw & 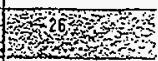 \\
\hline 57 & $88 \%$ & 65 & $70 \%$ & 25 \\
\hline 5 & $190 \%$ & 3ysts & $443 \%$ & (x) \\
\hline 54 & $98 \%$ & 56 & $38 \%$ & 32 \\
\hline $\mathrm{Hu}$ & $3 \mathrm{sp}$ & $5 \%$ & D. & 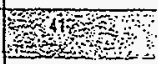 \\
\hline 38.3 & $101 \%$ & 50.3 & $28 \%$ & 44 \\
\hline \%o $501+$ & $30100 \%$ & 450 & sinots & $3{ }^{3}, 40$ \\
\hline 48 & $100 \%$ & 48 & $15 \%$ & 23 \\
\hline 34 & he & +1559 & $410 \%$ & $6_{12}^{22}$ \\
\hline 45 & $50 \%$ & 75 & $90 \%$ & $t$ \\
\hline 64442,4 & W joos & $\$<42$ & $8606 \%$ & $\sqrt{30}$ \\
\hline
\end{tabular}

SOTTWARE CATESOBIES

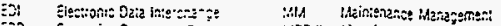

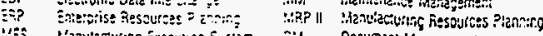

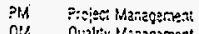

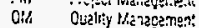

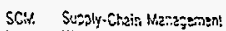
Sup:

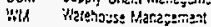

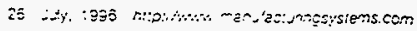
C-4 HNF-1539, Revo 


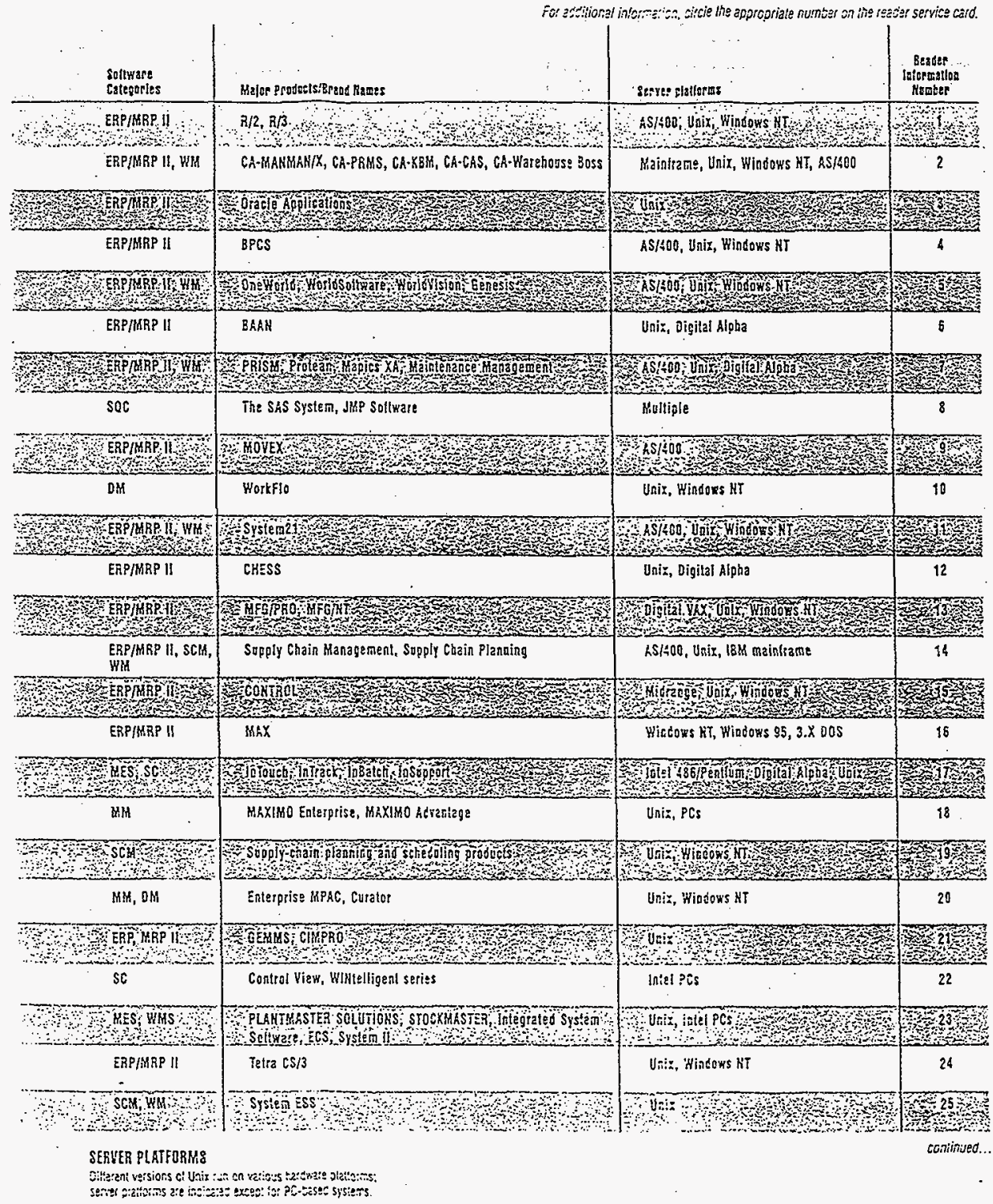




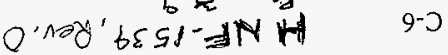

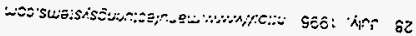

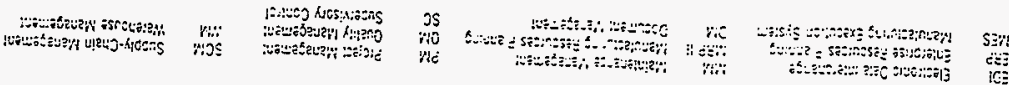

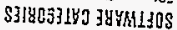

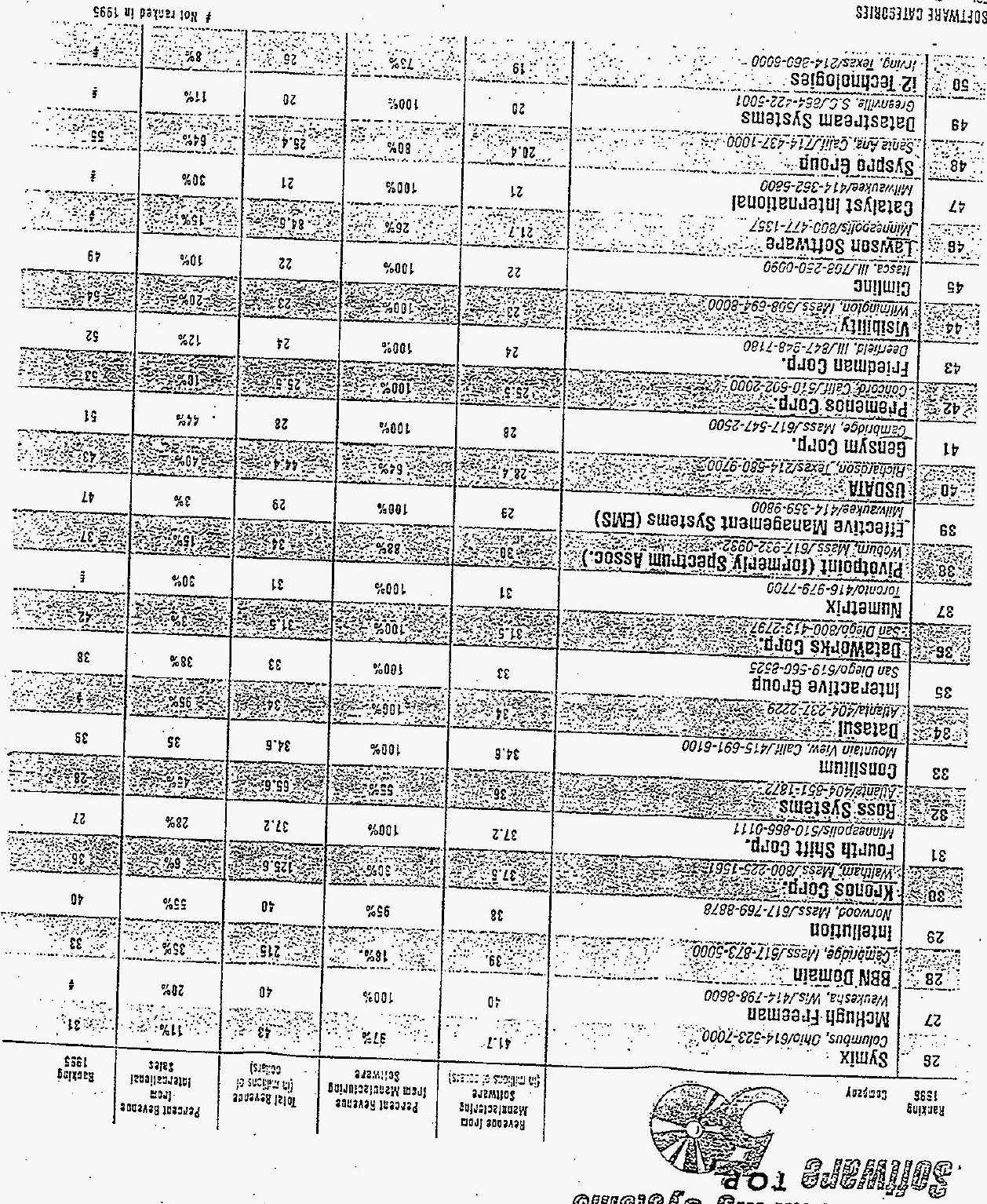




\begin{tabular}{|c|c|c|c|}
\hline $\begin{array}{l}\text { Etfware } \\
\text { catequeries }\end{array}$ & Major proctucts/Brate Hatat: & ntomation, circle the appropriate number on the & 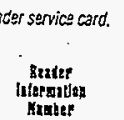 \\
\hline 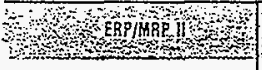 & 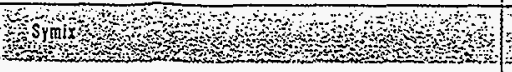 & 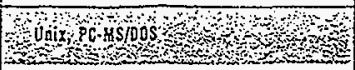 & 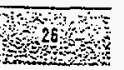 \\
\hline with & ont Plus & Unix & 27 \\
\hline Sy & 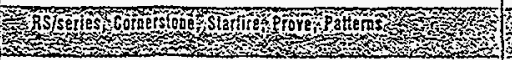 & 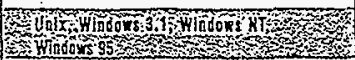 & Fis \\
\hline SC, MES & FIX HUL, FIX, FIX BOSS, PLART TV & Hindows, $3 . X$, Wintows NT, Windors 95 & 29 \\
\hline 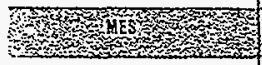 & 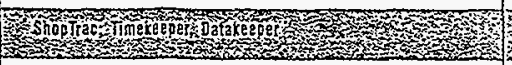 & W & 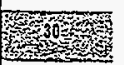 \\
\hline ERP/MRP U & Manufacturing Soltware System & intel-based platiorms & 31 \\
\hline 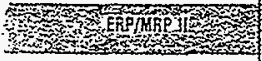 & W & 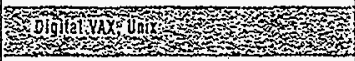 & 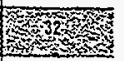 \\
\hline MES & HorkSlream DFS, Flowstrean & Unix, Digilal uus & 33 \\
\hline 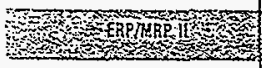 & 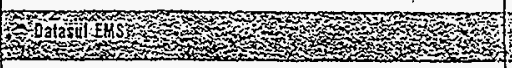 & Uno & $34+3$ \\
\hline ERP/MRP II & Intoflo, JIT Ealerprise Syslem & Uniz, Hindows MT & 35 \\
\hline W & F & yning & 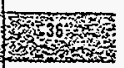 \\
\hline SCM & Supply-chais planaing and schedaliag prodects & Heltipie & 37 \\
\hline \% & 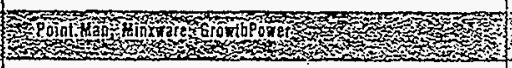 & 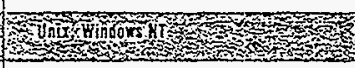 & 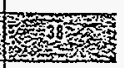 \\
\hline ERPIMRP H, MES & TCH-EHS, TCM-HES, TCH-SFIS & UDix, Hindows HT & 39 \\
\hline FA, & 1 & QX & 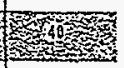 \\
\hline SC, MES & $62 \vdots$ & Unix, Hindows AT & 41 \\
\hline 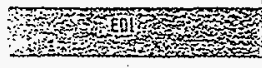 & S & 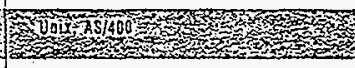 & 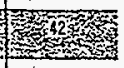 \\
\hline ERP/MRP II & WFA To-Order Hanufacleriag Systern & AS/400, Windows & 43 \\
\hline 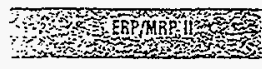 & I & 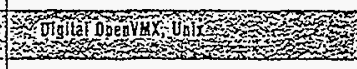 & ing \\
\hline QM, MES & Cimline Business Process Exetution Solvtions & Uniz, Hibcows HT & 45 \\
\hline Hos & 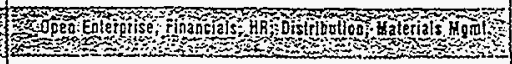 & 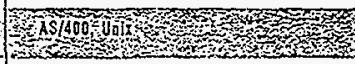 & 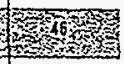 \\
\hline WH. & Catajyst wits & Unix & 47 \\
\hline 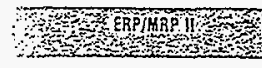 & 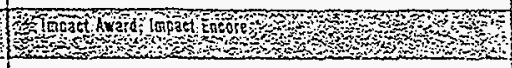 & 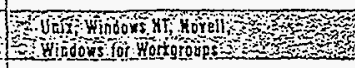 & 348 \\
\hline$M$ & MP2, Maintainll & Unix, PCs & 49 \\
\hline 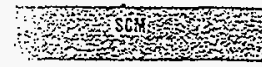 & 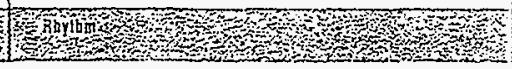 & 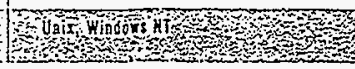 & sis \\
\hline
\end{tabular}

GERYER PLATFORME

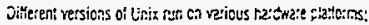

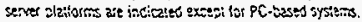




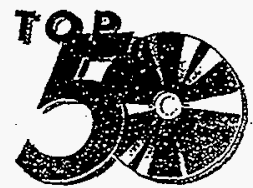

\title{
A fap-peaching impact
}

\author{
Microsoft Windows NT gains favor with supervisory-control vendors, users \\ Jim Fulcher, Senior Editor
}

0 ver the past several years, Microsof Windows NT has figured prominently in the proluct development plans of process-automation and control vendors. Of those vendors profiled in this section. one rocently received a tochnical inoovation atard from Microsoft, while another has gone so far as to declare Windows 95 and NT to be the environment of the fuoure.

Driven by an awareness of the increasing functionality of Microsoft's products, not to mention customer demand for Microsoft-compatible applications, supervisory-control vendors have been on the Microsoft bandwagon for quite some time. It's been big business, and there are no indications it will slow down anytime soon.

Supervisory-control systems-onginalily conceived of as PC-based operator interfaces for processes controlled by programmabic logic controllers (PLCs) have evolved fat beyond process monitoring and data acquisition. Today. applications include managing producuion and mantfactura ing execution.

As has been seen in numerous other technology marker segments, as microprocessor power becornes more readily available, the cost of the systems decreases. By combining reduced prices with richer fonctionality, new supervisorycontrol applications continue to arise.

In industrial-control applications, PCs typically have been used for operator interfaces, controller configuration. and data acquisition. Citing a lack of robusiness, reliability, and security. manufacturers historically have been hesitant to use RC platforms for mission-critical applications.

That's where Microsoft entered the picture. Once some initial bugs were ironed ouc. Windows it has caused many manufacturers to reevaluate their concerns about PCs and the shop noor. NT's multitasking. high leveis of security. and memory and crash protection have done much to soothe those worries. And with Windows. Windows 95, and other
Microsoft products continuing to set the standard for office applications, nsing Window's NT for the shop floor makes sense because it naturally integrates with those other applications.

The first four companies profiled-Wonderware. Rockwel], Intellution, and USDATA-are the most soccessful companies in a very successful market.

The fifth company profiled. Gensym, is one of the suceessiui vendors of what has been called "arrificiat inteiligence" in production environments. When used in manufacturing, Gensym's "intelligent real-time" system's software is most ofien used to monitor and manage production processes.

\section{Wonderware Corp. \\ Some growing pains}

After sailing along smoothly since launching its In Touch supervisory-control package in 1987, Wonderware Conp. appeared to hit rough waters last year. Despite a 54 -percent increase in reveaue. the company closed its books on 1995 with a net loss of $\$ 14.3$ million. Its stock price flucanued wild $y$ as well, rising as high as $\$ 42$ and falling belo=n $\$ 13$ over the course of the year.

Despite these outward appearances, a close look inside Wonderware's Irvine Calif., headquarters reveals not so much a company in dire straits, but one working to escablish a new jdentity. What we've experienced this past year is typical of what happens when an entrepreneurial company reaches critical mass and is forced to grow up." says Don Allen. Wonderware's director of public relations. "This is our adolescence.-

In other words, recent management changes at Wonderware are not much different than what bappens al any fast-rising 
startup that becomes a mature corporation. Last November, Wonderware's principal founder. Dennis Morin, retired as chaiman and CEO. Both tities. as well as the muntle of president, are now held by Morili's hand-picked successur, Roy Slavin, former CEO of Sienens Industrial Automation.

In true adolescent fashion. Wonderware no longer wants to be seen as a company dependent on a single product. Instead, it wishes to be known as a first-rale provider of an integrated suite of factory automation tools. To that end, the

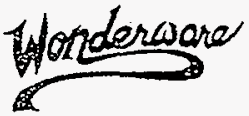
company has unveiled The Wonderware Factory Suite, a group of five produets designed to control and exccuse manufacturing processes, in addition 10 aiding in the maintenance and repair of manufacturing equipment.

Allen says Wonderware's original management team had long dreamed of taking the company in this direction, "but they also knew they didn't have the big company experience to pull it off." Thus, the selection of Slavin to head a new management team. Allen says Morin felt the combination of Slavin's experience at nunning a large industrial automation company and the stilis he exhibited during several years he spent acquiring and ouming around troubled companies would prove handy in leading Wonderware into a new era.

The original management team actually started Wonderware on its present course in 1994, when it purchased Rubicon Technology of Mountain View, Calif., and its product for automatic troubleshooting and repair of manufacturing equipment. That was followed by the June 1995 acquisition of EnaTec Software Systems, of Cupertino, Calif., developer of a manufacruring execution system (MES). Two months later, Wondenvare bought York, Pa.-based Soft Systems Engineering (SSE) and its product for controlling batch-production processes.

These were all logical moves for a company seeking products to complement its highly successful InTouch package, but Allen says Wondentare's goal was to make these products fit a common architecture. Once that was done, he says, it was time to amnounce the Factory Suite. The suite consists of the following products: InTouch, Wonderware's original supervisory-control package; InTrack, an MES component based on the product purchased from EnaTec: InSupport, an automatic equipment troubleshooting and repair package derived from the Rubicon Technology system; InBatch, a system for modeling and controlling batch-oriented production processes modeled on the system acquired from SSE; and DDE Servers, inputoutput devices that facilitate data sharing between InTouch and factory-floor control devices. Allen says Wonderware now is working to make all of the products compatible with a plant-floor database product called Prealism that was purchased last December from Profes- sional Technology Management Lid. of South Africa. Despite all the changes, there is one part of its original heritage that Wonderware wants to keep: its identification as a Windows-based solution provider. Much of InTouch's original success has been attributed to its starus as the first industrial application to run on Windows. The Factory Suite is designed to run on Microsofi's BackOffice family of server products. And Allen says Wondenware hopes to take the Microsoft tradition a step further. "Eventually, we want to offer the entire Factory Suite on a single $C D$, and sel] it the same way Microsoft sells BackOffice," Allen says.

If that strategy works, Wonderware's eamings and stock price, both of which already have begun to rebound, should continue rising for some time to come.

\section{Rockwell Software}

\section{On a virtual roll}

Although it happened less than two years ago, the creation of Rockwell Software from the merger of the Allen-Bradley Co.'s software business with ICOM Inc. seems almost like ancient history. That's mainly because the melding of the two companies' management structures and product lines was a fairly smooth process.

The company now is neatly organized into four strategic business units. The Programming unit is responsible for products used to program PLCs and related equipment; the MMI group handles supervisory-control and data-acquisition products; the Communications group develops software to facilitate data sharing between shop-floor control and higher level systems; and the Components

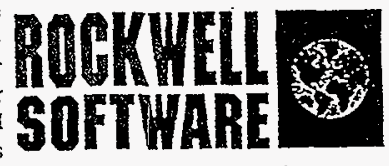
group creates specialized packages that manufacturers can use to develop their own custom applications.

With this structure in place, Rockwell Software is concentrating on maintaining its leadership position in the process-automation marketplace. That has generated tremendous activity on the "virtual campus" created through electronic links beiween the company's dual headquarters in West Allis, Wis. and Highland Heights, Ohio.

Each Rockwell Software business unit has released at least one series of new products within the past year, all of them designed to run in the Windows environment. "We believe Windows NI and Windows 95 is the environment of the future," says Mark Moriarty, Rockwell Software's commercial marketing manager, in explaining the new product blitz. "That's where we have to be if we want to stay leadingedge and give customers better tools to do their jobs." 


\section{Supervisory control}

Moriarty says Rockwell Software is particularly proud of the new products from the Components and -MMI groups. The MMI products are led by a package called RSView, a supervisory-control system designed for the Windows NT and Windows 95 platorms. The features in RSView include an alarming function that allows the user to monitor process incidents, trending capabilities for real-time plotting of process-information trends, and an event detector that triggers system commands based on the occurrence of certain events, which can be defined by the user. RSView is complemented by another new MMI group product, RSTrend. This package displays real-time and historical data sirnultaneousiy, expanding the capabilities for process monitoring, data analysis, diagnostic troubleshooting, data reporting, and even regulatory compliance.

The Components group released a family of products under the name RSTools. These products, built in compliance with Microsoft's ActiveX component architecare, are specially designed software packages that allow users to create custom applications using Microsoft development tools like Visual Basic and Access. RSTools include programs for creating everything from custorn gauge controls to special data comparison applicarions. Because they are based on the ActiveX rechnology, Moriarty says, programs created with RSTools can be made to freely interact with applications not created by Rockwell Software.

The Rockwell Software Comnunications group has a new family of products, called RSLinx, that facilitates data sharing between all Rockwell Software applications as well as third-party applications that use Microsoft's DDE utiliry. The latest releases from the Components group are Windows-based programs for programming Allen-Bradley SLC 500 and MicroLogix processors.

Moriarty says the underlying structure of all these products allows manufacturers to, in effect, construct their own process-automation networks. He says it also proves that Rockwell Software is "not focused on just providing products, or groups of products. WWe are looking to provide solutions."

\section{Intellution}

\section{Just rolling along}

Though it has been part of Emerson Electronic for more than a year, not much has changed at Intellution. The Norwood, Mass., company continues to chum out leading-edge processautomation and control software, and manufacturing companies around the world keep gobbling it up.

Intellution sells a complete suite of factory automation and control software. The suite starts with the FDX package, a clienvserver system that provides everything from data collection to monitoring and control of production processes. The system provides graphical displays for things like historical trending, and has various options for setting alarms to aler production managers of situations that warrant their attention. Other products in the suite are FIX MMI, a man-machine interface product for viewing production operations and conditions; FIX BOS, a recently released product for monitoring and controlling batch-oriented production in the process industries; and PLANTTV, a graphics-based system that lets plant managers check the status of any operation within an organization, even at remote sites, at any given time.

Intellution posted record revenues in the second quarter of this year, a 44 percent increase over the same period last year. Those revenue numbers were fueled by a 50 percent increase in product shipments over the second quarter of 1995 , with

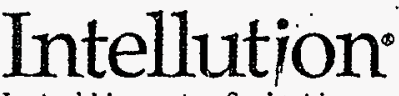
Inteliggent Solutions

the latest release of FIX leading the way. The market also has responded well to the December 1995 release of FIX BOS.

James Blaschke, Intellution's vice president of sales and marketing, attributes Intellution's current success to "the market's rapid acoption of 32-bit computing environments." Thirty-two bit computing is a synonym for Windows NT and Windows 95. Intellution began building its products for those environments in 1993, and it appears customer demand for those systems finally has materialized. Blaschke says Intellution's latest releases "have not only exceeded our revenue and profit goals, but they bave brought on accolades from our peers and the press."

The accolades include Intellution's recent selection as winner of Microsof Corp.'s Leonardo Medal for Technical Innovations in Manufacturing. The award was based on implementation of a process-monitoring and control system at a Chevron plant that combined Intellution applications with Microsoft products, including Microsoft's Baci-Office server suite.

Intellution president and CEO Stephen Rubin says the award "solidifies our leadership role in the industrial automation software market, and we intend to extend that leadership by continuing to convert technology into world-class manufacturing solutions to enhance our customers' comperitiveness."

\section{0 uspata corp.}

\section{Growing in all directions}

Any way you look at it, this has been a year of growth for Richardson, Texas-based USDATA Corp.

The pattern staned last August, when its ownership base was increased through an initial public offering. The money raised by selling those first shares of stock was then used to augment nearly every phase of the company's operations. 
The product development budget grew by 40 percent, with much of those funds used to complete a major expansion of FactoryLink, USDATA's flagship factory-automation toolset. Forty new people were hired to provide additional technical suppor for the expanded product line and to staff two new worldwide support centers-one at the Richardson headquarters and another in Europe. USDATA also heightened its public profile through greater use of advertising and more aggressive marketing strategies, including formal marketing alliances with companies like Microsoft, Sun Microsystems, and Siemens Industrial Automation. Finally, the company increased its sales staff and started placing more emphasis on intemational sales.

While all these moves are critical to the future of USDATA, the one that probably is most important to its customers and potential customers is transformation of its FactoryLink product. In keeping with its new high-profile marketing style, USDATA unveiled this product at a joint press conference with Microsoft at the National Manufacturing Week trade show in Chicago this past March. Called FactoryLink ECS, it figures to broaden USDATA's market.

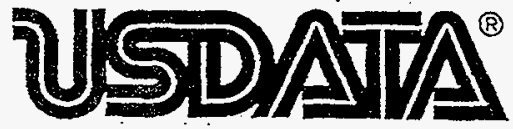

Up to now, FactoryLink has been used by companies seeking to build supervisory-control and data-acquisition systems. But with the enhancements included in FactoryLink ECS, the product can be used to build and control manufacturing execution systems as well.

John Keenan, USDATA's vice president of worldwide marketing, says FactoryLink ECS is a reflection of manufacturers' desire to "move away from automating single functions, and look more toward corporatewide, or enterprisewide, control architectures." Keenan says USDATA. focused on four key areas in making sure that FactoryLink ECS would, indeed, allow users to build systems that could be deployed across an enterprise. These areas are: systern power, ease-of-use, compatibility with multiple operating platforms, and a strong USDATA support organization.

The most exciting upgrade from a technical standpoint relates to ease-of-use. USDATA addressed that issue by creating a set of tools it calls Power Dragons.

These essentially are application objects that users can pull from a USDATA object library, or create for themselves using the FactoryLink software. They then have the ability to reuse the objects-by dragging and dropping them-to expand an existing application or begin creating a new one. Keenan says early studies have shown that use of Power DragOns cuts typical application-development time in half.
While it expects big things from FactoryLink ECS, Keenan says this product is just one part of USDATA's strategy for offering enterprisewide automation systems. USDATA also offers a warehouse-management system called Traklink and a warehouse application-development tool called VisualLink, in addition to selling automatic data collection hardware.

Keenan says the company plans to combine FactoryLink ECS with its other products "to offer an integrated suite of software tools, applications, hardware, and services for companies looking to build complete manufacturing and distribution systems."

\section{Gensym Corp.}

\section{Enterprise control}

Sometimes its difficult to tell whether Gensym Corp. is a commercial software developer or a private research laboratory. That's because the Cambridge, Mass., company consistently finds practical uses for a class of technology that shows up more often in science fiction movies than it does in the business world. Gensym's entire product line is built on the use of expert systems, or as Gensym's president, Dr. Richard Moore, prefers to call them, "intelligent systems."

Gensym's systems start with its flagship G2 package, which really is more an environment in which users employ intelligent software to build their own systems for autornating and controlling production processes. Most $G 2$ users build interfaces for controlling shop-floor systems or production processes, but it also has been used to create manufacturing execution systems. $G 2$ is supported by a variety of products that not only allow users to execute and control produccion processes, but optimize them as well.

The supporting products include G2 Standard Interface, a communications program that facilitates smooth passing of data between various applications and databases; Telewindows, an interactive interface program that lets multiple users view and work with $\mathrm{G} 2$ applications simultaneously; G2 Diagnostic Assistant,

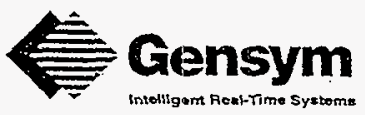
a system that allows production workers to build applications to improve their work processes; and Dynamic Scheduling Package, a program for creating and adjusting schedules of complex operations. A new product from Gensym, NeurOnline, offers an environment for building automated production processes with neural-network technology.

Gensym also is taking its technology to the internet with two new products called G2 WebLink and G2 WebMiner. WebLink provides a means for users to access and use the tools available in the $G 2$ environment through World Wide 


\section{Attachment 4: PLCs Plus Internal Survey}

The following data resulted from a survey conducted internally at the offices of PLCs Plus, polling several of the systems integrators on staff:

\begin{tabular}{|c|c|c|c|c|c|}
\hline $\begin{array}{c}\text { ATTRIBUTE } \\
\begin{array}{c}\text { System } \\
\text { Requirements }\end{array}\end{array}$ & WW & GFW & FIX & DACS GFW & DACS FIX \\
\hline Operating System & $3 \star$ & $2+$ & 3 & 3 & 3 \\
\hline Ease of Learning & 4 & 2 & 3 & 3 & 2 \\
\hline $\begin{array}{c}\text { Help/Tech } \\
\text { Support }\end{array}$ & 5 & $4 / 3$ & 4 & 4 & 4 \\
\hline $\begin{array}{c}\text { Graphic } \\
\text { Development }\end{array}$ & 4 & 3 & 4 & 4 & 3 \\
\hline $\begin{array}{c}\text { Tools/Scripting } \\
\text { Trending }\end{array}$ & 3 & 4 & $3 / 4$ & N/A & N/A \\
\hline Drivers & 2 (DDE) & 3 & 4 & 4 & 3 \\
\hline Data Sharing & 3 & 3 & 4 & N/A & N/A \\
\hline $\begin{array}{c}\text { Flexibility } \\
\text { (Expansion) }\end{array}$ & $*$ & Keys/Driver & $(*)$ & N/A & N/A \\
\hline Networking & 2 & 3 & 5 & $4 / 5$ & 4 \\
\hline Alarming & 4 & 3 & 4 & N/A & N/A \\
\hline Reliability & 4 & 2 & 5 & 3 & 5 \\
\hline
\end{tabular}

Notes:

Scale: $5=$ Best, $1=$ Worst

$\star$ - No O.S. portability

* - If keep strategy

+ - No NT / RTS-DOS

* - J/O pts internal, Drivers separate, internal blocks count

$\left.{ }^{*}\right)$ - I/O pts, drivers separate $\}$ scalable

מ - If ACRNET

While "surfing" the web for "Hits" related to the four software packages, the following was obtained:

Wonderware - 7,300 Intellution-3,950 Iconics - 258 CiTect -256

D-1 HNF-1539, Rev. 0 


\section{Competitive Review - Paragon TNT \\ July 15, 1996}

\section{Overview}

Paragon TNT is from Intec Controls - Walpole, MA. Paragon was first introduced as Paragon Control in 1986 as a DOS based package. The name was later changed to Paragon 500/550 around 1990. Control and 500/550 were DOS based only and there are approximately 8,000 DOS packages installed worldwide.

Paragon TNT came out in 1993 as OS/2 only. In late 1995 it was released on Windows NT and just recently it was released on Windows 95 . There have been approximately. 3,000 Paragon TNT packages sold with about $75 \%$ of them being OS/2. Paragon was never released for Windows 3.1 or Windows for Workgroups.

\section{Paragon TNT Architecture}

The Architecture is broken down into 3 major server subsystems and a Client subsystem: Process 1/O (PIO), Continuous Strategy (CS), Data Manager (DM) and the Operator Interface (Ol) client. The 3 servers and the OI client communicate to each other via the Common Resource Access. This CRA is the proprietary backbone that allows the communication between the servers and client. The CRA can be distributed over a network. This architecture is what Intec calls Functional Software Architecture (FSA). FSA is not an industry standard, the name was made up by Intec.

The 3 servers can be on one node or distributed over a TCP/IP or NetBios. In both the standalone application and networked application each server has its own configuration and database files. You cannot add blocks to the database files and have those changes start scanning. The server must be brought down and restarted before any changes start scanning. In order to pass data from the PlO server to the CS server you must import (duplicate) the PIO database into the CS database.

\section{Process //O Server Subsystem}

The PIO server allows the communication to PLC's and control devices. The server uses TNT Engines (Drivers) for the control. Intec writes the major portiorl of their drivers and they do not promote third party drivers. The driver list is limited to the common PLC's and control devices: Allen Bradley, Modicon, Opto, etc. 
The PIO provides:

- Signal Conditioning

- Alarming with 4 different alarm levels (LoLO, Lo; Hi,HiHi).

- Block read/writes

- Diagnostics.

There is a driver development kit, however, there is no training offered for this toolkit.

\section{Continuous Strategy Server Subsystem}

The CS server executes the continuous control of the process. The library includes math, Boolean, discrete \& supervisory control, and ASCIl string functions.

This server is the heart of Paragon. It has a graphical interface for connecting blocks. The database has a layering concept. Function blocks are grouped together inside process blocks. There is a limit to how much each process block can hold that is based on the type of function blocks.

Function blocks are linked together with a connecting line and process blocks are linked together with a conduit. Each interprocess connection inside a conduit must be configured. Interprocess connections must also have terminators. Here is an example: Make one connection from an Analog Input Block in Process $A$ to a Math Block in Process $B$ and run it. First configure the analog input block and a terminator block, then draw a line from the analog input block to the terminator block. Now inside the other process block, configure the math block and another terminator and draw a line from the math block to the terminator block. Now draw a conduit from process $A$ to process $B$ and configure the conduit to connect the two terminators. In order for the two new blocks to start scanning you need to first save and compile the database, and then restart the server. For large databases this compile can take up to 15 minutes.

The majority of the CS function blocks are continuously scanned at a scan time. Also there is no timer block in the CS. To measure time you need to build a chain of 3 blocks.

When TNT was first released and until the NT version, the CS was still a DOS application. 


\section{Data Manager Server Subsystem}

The Data Manager server stores historical and real-time data from the CS and PIO servers. The DM is also a place holder for all alarms and system, and operator events.

Each storage area (block) must be configured using the Data Manager Builder, and like the CS you cannot add block on scan without restarting the server.

The Relational Database Interface is also a function of the DM. There is a SQLODBC interface, however the building of the SQL commands is very difficult and there are no short cuts for entering the commands. Intec provides ODBC drivers for their OS/2 version. The list includes Dbase, DB2/2/, Oracie, SQL Server and Text. For the NT and 95 versions you must provide the driver. On NT and 95 intec does not publish any testing with any Database or driver. There have been some problems with even the Microsoft 32 bit ODBC drivers:

\section{Operator Interface Client Subsystem}

The Operator Interface Client is for creating and running the displays. With the Of you can provide Data Display \& Entry Boxes, Real Time and Historical Trends, Alarm and Event Journals, Bitmaps and Presentation Manager GUI Objects such as List Boxes, Radio \& Push Buttons, and Sliders. Animation of objects is also supported by the $O$.

The bitmaps do not become a part of the display and are very slow in opening a display. The alarm summary journal has no color or blink configuration for the Text messages. The Library of common valves and pumps is very limited.

\section{String Handling}

ASCII string data collection is done directly into the CS and can bypass the PIO. While Intec promotes being able to collect ASCII data without an $\mathrm{V} / \mathrm{O}$ driver as a feature, if the string messages are short and do not require any acknowledgment, this is a nice interface. If the string messages are complex you need to develop a driver to handle the $1 / 0$.

All string blocks are event based. 


\section{Microsoft 32 bit compliant}

TNT has only been on a Microsoft Windows platform since late 1995 and only under NT and 95 .

Intec came out with the NT release first and shortly after that they released the 95 release. The first 95 release was actually separate code. There is no documentation stating that Paragon runs as a service under NT.

\section{Miscellaneous Information}

The DDE link is a client link and there are no macros that come with the package. There is also no recipe builder or SPC. Redundancy configuration under TNT is also a problem. There is no published write up detailing any redundancy for Paragon TNT. Lastly, the DOS release 500/550 cannot interface to TNT. Paragon TNT uses a Rainbow protection key for copy protection.

\section{Pricing}

Paragon TNT is broken down into builder and enablers for runtime functionality. To develop a 256 point networked node with DDE, Alarm, Trending, one Driver and all Subsystem Builders the price is $\$ 9,500$ list. The same configuration as a Stand Alone node would be around $\$ 6,900$ list. This configuration as unlimited $1 / O$ points would be an additional $\$ 1,800$. (Contact your Inside Sales Engineer for a Price List).

\section{Summary}

Paragon TNT for Windows NT and 95 is just about a year old and the driver list is very limited as well as the functionality compared to FIX.

Intellution's Inside Sales Department has a complete literature package with pricing for Paragon TNT, along with a full and limited demo package with documentation.

- Intellution Inside Sales. 


\section{Paragon TNT Price List}

U.S. Pricing - Effective with V3.2 Release

\section{How to Specify Your Order}

For each computer (stand-alone or network node) which will run Paragon TNT, you must specity one of the following combinations of TNT products:

- Some number of Enablers and one or more Builders (a Full System)

Some number of Enablers only (a Runtime System)

$\because$ One or more Builders only (a Builder System)

Keep in mind that you can run only one TNT product at a time on a single computer.

Enablers:

. Enablers determine the number of Runtime TNT Clients, Servers, and related Options you can run concurrently on a single computer: Each TNT Client; Server; and Option requires a specific number of enablers: You must purchase at least the total number of enablers required to activate all of the Runtime Clients, Servers, and Options your application requires. Note that whatever the number of enablers you purchase, you will still receive all of the available TNT Clients, Servers, and Options. The enabler system allows you to change the configuration of your TNT package before system start-up by exchanging one Client, Server, or Option with another of equal or lesser "enabler value". For example, you could enable a Process $1 / O$ Oriver (3 enablers) for a particular application, and then later disable the diriver and enable the DDE Client Link (2 enablers) for a different application. Enablers are sold in bundles called "EPAKs", specifying the number of enablers required, e.g., EPAK32 (see next page).

\section{Builders:}

Builders are the tools required to build specitic applications for each of the TNT Clients and Servers. You must specity in your order which Builders will be required for each computer. Builders are not governed by enablers, i.e., you cannot "disable" a builder and enable something else:." Each Builder is available in two different versions: Builders for stand-alone applications, and Builders for networked applications. An application built with a networked Builder will run in stand-alone mode, but applications buitt with a standalone Builder will not run in a networked environment.

\section{Pricing Examples}

The following pricing examples describe the amount of enablers and types of Builders required for a stand-alone application and a networked application. Please see the.next page for a listing of all Clients, Servers, and Options, their enablers, and prices.

\section{Example $\# 1$}

The application requires a stand-atone graphical operator interlace which acquires and oisplays tala Irom 60 VO poins onginaling from a single brand of PLC. Trending and data togging is also required.

Fequirect Subsystemsioptions

OI Cperalos Interface Client

Pl0-64 Process VO Clierd - 64 points

PIO-D One PIO driver.

OM Data Manager Server

DUS-HST History Option

For a Runtime-only System, order:

Tolal Required: 11 enablers

* OlEnablers

4 enablers

Q enablers

3 enablers

O enablers

EPAK11' $=\$ 2200$

\section{Suildars Reguired dor Developenent}

Ol\&-S Operator Interlace Builder. Siand-abone

Prog-s Process VO Builder - Stand-abone

DME-S Data Manager Builder - Stanc-abne

Price
$\$ 700$
$\$ 350$
$\$ 350$
$\$ 1400$

Full System Order:

EPAKII' C $\$ 200+$ Buildare o $\$ 1400=\$ 3500$

'While this EPAK would be sutliciert to run the application, we strongty advise that "extra" anablers be putchasod to allow for untoresoen application fequiremants.

\section{Example \#2}

The applicalion requires a futy nelworked TNT node with lull operalor graphics, acoess to several hundred to points from a PLC neiwork, trending and cala loging. math computations on acquired dala. and the ablity to transter data to third-party applications via DDE.

soditionaisuosystemscolions

Ot Operator Intertace Client

PLO-512 Process VO Subsystem - 512 potnts

PIO-D On PIO chiver

Ds Data Manager Server

DA-HST History Option '".

CS Continuous Strategy Servas

DOE-L. DOE Client Link

NAON Nelwork ACcess Oplion

For a Runtime-ardy Syetem, order:

Eol Enables

4 enablers

6 enablers

3 erablars

0 enablars

4 erablars

2 anablars

2 enablers

Segablers

Total Requirof: 27 enablers

EPAK2T $=\$ 5400$

Builders Rosulted tor Dovaloment

Price

OIB-N Operaror interiace Builder - Network Apps

stoso

PKB-N Process VO Builder - Nelwork Apps

$\$ 700$

DMB-N Data Manager Buider - Network APDS

$\$ 7 \infty$

CSE-N Continuous Sralegy Builcet - Network Apps

$\$ 1052$

53500

Full Systom Order:

EPAK2T @ S5400+ Bulldort $\theta \$ 3500=\$ 8900$ 
Operator Interface client

Process graphic display and operator interaction.

Product Description

Ol. Operator Interface Client

Process ע/O Comms Server

interface 10 $/ / O$ hardware, basic signal conditioning and alarming. Select one server option and one or more driver options.
PIO-64 Process lOO Server - 64 Points
P10-256 Process 110 Server - 256 Points
PIO-512 Process $1 / 0$ Server - 512 Points
P10-2048 Process $1 / O$ Sever -2048 Points
PIOUL. Process VO Server-Unlimited Points
PIO-D. Procass VOO Driver (each PIO type - Intec or user developedy ${ }^{\circ}$

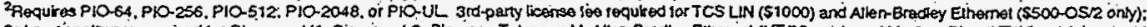

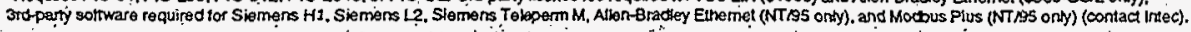

\section{Continuous Strategy Server}

Functions tor computation, supervisory and direct control, and string handling.

CS-1500 : Continuous Strategy Server - 1500. (maximum of 1500 function blocks). Includes Computation Blocks.

CS-UL Continuous Strategy Server-Unlimited (unlimited number of function blocks). Includes Computation Blocks.

CS-SUP. Supervisory Block Option?.

CS-STR String Elock Option

CS-UB User Block Runtime Link"

'Pequires CS-1500 or CS-UL. Reler to the back page for the tundion blocks contained in each option.

\section{Data Manager Server}

Data collaction and reduction, alarm and event management, relational database/SQL support, and historical and real-time 1rends.

DM-500 Data Manager Server-500 (maximum of 500 functions, from options below). Includes default alarmevent functions. 0

DM-UL. Data Manager Server-Unlimited (unlimited number of functions). Includes default alarm'event functions. . 3

DM-TND Trending Option. Real-time trending with compression.4

DM.HST History Option. Historical data collection and trending with compression.

- DM-AE Advanced Alarm/Event Maragement Option. Permits configuration of unique alarmvevent functions.*

DM-RDB Relational Databass interface Option. Exporflmport data tofirom OOBC-compliant RDB systems."

DM-AOV Adyanced Linkable Functions Option.: Set of linkable data manager functions."

Hequres DM-500 or DM-UL. Reter lo the back page tot the lunctions containod in each option.

\section{Recipes Client}

Recipe download/upioad functions.

RECP . Recipes Client

\section{Engineer Interface Client}

Application test interface.

El Engineer Interface Client

DDE Client Runtime Link

Third-party application software link via DDE.

DDE-L DOE Client Runtime Link

Client Object Interface (COI) Runtime Link

Links user-developed or intec-written COI clients to TNT.

COl-L : User Client Runtime Link (for clients developed using COI or COK tools)

Network Access Option

Full notworking capabilities for a single TNT node.

NAO-N Netbios Network Access Option

VAO-T TCP/IP Network Access Option

ENABLER PRICING

Enablers are sold for $\$ 200$ each in Enabler Pak's (EPAK's). For exarmple, if you require 10 enablers, you would purchase an EPAK10 at a price of $\$ 2000(10 \times \$ 200)$; if you require 27 enablers, you would purchase an EPAK27 at a price of $\$ 5400(27 \times \$ 200)$, etc. For a Builder-only system, specily EPAKO. Be sure to inciude product code, e.g.,EPAK10:TNTOS2 (see below). Complete documentation is shippod with each TNT system that includes builders. For EPAK only orders, only the User's Guide is included.

\section{PRODUCT CODES}

Please include the appropriate Product Code with each Product Number, e.g., EPAK32: TNTOS2 or UPY:550TNTNT.

CNTRL - Paragon Control 550 -Paragon 550 TNTNT Paragon TNT tor Windows NT

500 - Paragor 500 TNTOS2 - Paragon TNT for E-6

TNTW95 - Pararen TNT in. IAlimat.... n.

HNF-1539, RON 0 
Builders:

When selecting Builders, select either all-Stand-alone Builders or all Networking Builders. Networking Builders are required for LAN * networking. Different Builder types cannot bé mixed on a single computer. For "Builder-only" systems, specify EPAKO on order.

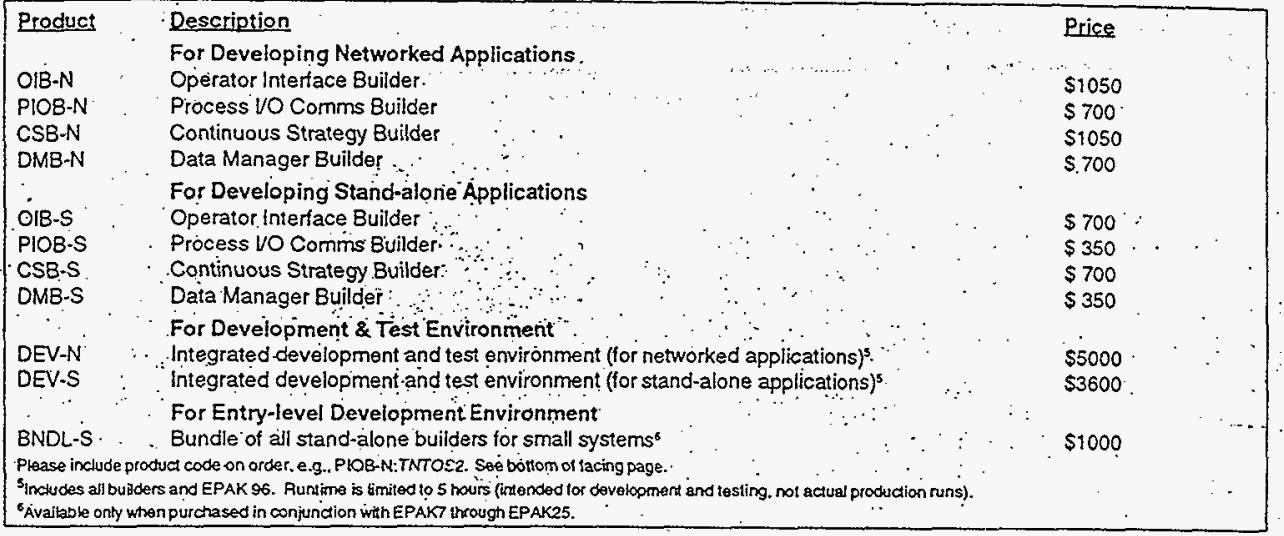

\section{Developer Kits}

TNT Developer Kits include all of the software toois necessary to develop and integrate custom functions for Paragon TNT.

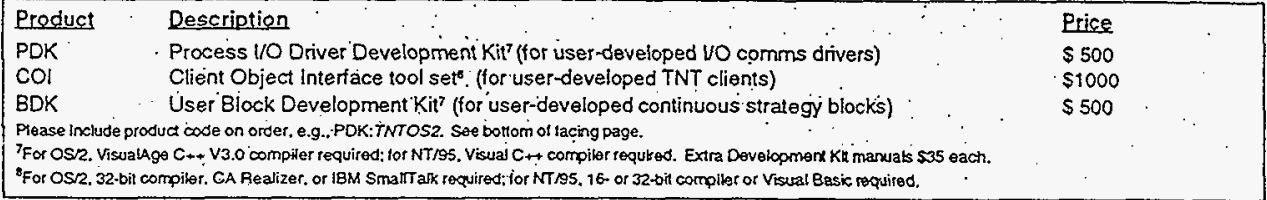

\section{COl-based Client Applications"}

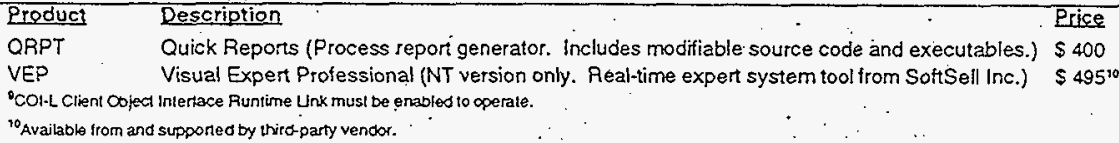

\section{Support Programs}

The Customer Suppont Program (CSP) includes telephone support, no-cost version updates, and other priority services for a period of one year. The CSP contract should be purchased within the 90 day warranty period to avoid a surcharge. (Note that sotware version updates are only available to customers with CSP.) An Upgrade is the exchange of one Paragon product for a ditferent Paragon product. Note that adding additional enablers or builders to an existing TNT product is accomplished through an Upgrade (UP1). Corporate Training is held at a facility selected by the customer; Regional Training is held periocically in Intec-selected cities throughout North America.

\section{Product}

CSP:(prod, codeJ

UP1:(existing prod. code/new prod. code)

UP2:(existing prod. code/new prod. code)

UP3:(existing prod. code/new prod. code)

TR-INTEC:(prod. code)

TR-INTEC-ADV:(prod code]

TR-CORP:(prod, code)

TR.REG: (prod code]

DOC:(prod code)

SDX

OSS

$A C$

"Site discounis avaidable.

${ }^{12}$ Delia list cannot be less inan zero. UP3 requires exctange of protection key. Please spocity package serial number and condiguralion of existing product

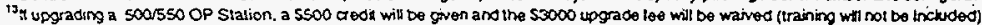

${ }^{14}$ Additional anendees at 5250 each.

\section{Description}

Customer Support Program for 1 year (per product)

Upgrade existing. TNT product within same operating system

Upgrade existing TNT product to a different operating system

Upgrade Paragon CNTRU500/550 to TNT

4 Day Training (Part I) at intec (1 computer per person)

4 Day Training (Part II) at Intec (1 computer per person)

4-Day Corporate Training at customer facility (max. of 6 people)

4 Day Regional Training (1 computer per person, min. of 8 people)

Specity Ref: Manual, User's Guide. PIO Engines, and/or Tutorial

Same Day Express Shipment (order placed betore 3 PM EST)

On-site Support (per day)

Application Consulting, per hour (telephone) or per day (on-site)

\section{Price}

$12 \%$ of List 11

$\$ 450+$ dehta list\$ 12

$\$ 450+$ dehta list\$12

$\$ 3000+$ dehta list\$\$2.13

\$1495/person

$\$ 1495 /$ person

$\$ 10,000+$ expenses ${ }^{14}$

$\$ 1895 /$ person

$\$ 65$ per manual

$\$ 200+$ treight

Contact intec

Contact intec 


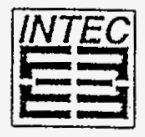

The World's First Functional Software Architecture Paragon TNT

A White Paper Prepared By:

Intec Controls Com.

55 West Street

Walpole, MA 02081

Tel: 508-660-1221 Fax: 508-660-2374

August 10, 1994 


\section{Traditional Package Approach to Software Design}

Software design' philosophy over the past decade has primarily focused on an approach that treats the entire application set as a single software package with a single common database. Automation software packages developed using this design'were intended to operate as stand alone entities in a single hardware platform, very often a PC, with a monolithic real-time database. All required automation functions typically accessed this "tightiy coupled" local database.

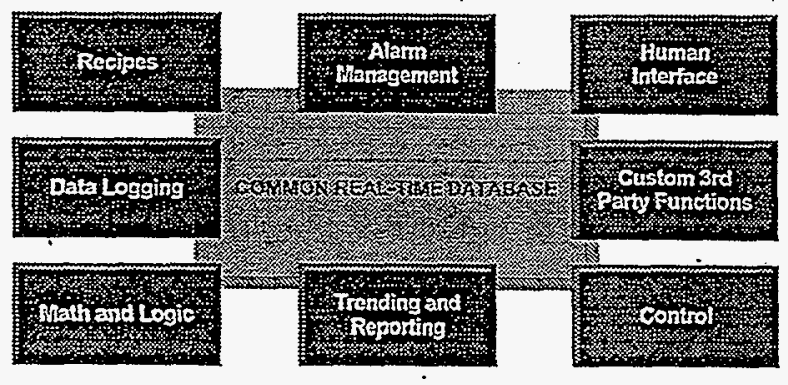

Traditional, Monolithic Design

In recent years customers have required larger, more distributed solutions. The response to this requirement by the software package vendors has been simply to network a number of computers together, each with its own package and a copy of the database.

The simpliny ol design of the packaged approach has been appealing; but as requirements have growin more demanding the inherent constraints of packaged approaches have surtaced. Automation system capability has been constrained by the limitations of the hardware platform the packages operate on. Most packaged software is designed on a "one size fits all" basis, therefore expanding packaged systems typically requires purchase of an additional complete package even if only limited incremental functionality is needed. Scalability and effective expandability of traditional packaged automation systems has been quite limited. Reliability has also been a significant issue

- with automation software packages. The monolithic design of these systems limits reliability, with a single fauit often causing the entire system to fail and/or cause the database to be corrupled. Finally, automation software packages have typically been designed around single operating systems. When the operating system encounters the end of is life cycle, the automation software package follows suit. 


\section{What Are the User Requirements?}

Over the past few years, users of software automation packages began to encounter the constraints imposed as a result of this packaged approach. Vendors were driven to investigate new design approaches to overcome these constraints. Users demands inciude meeting a number of new software requirements:

- A functionally modular design which gives them the freedom necessary to match their application requirements with the automation solution.

- Hardware independent software to run on a variety of hardware platforms and in a variety of software environments.

- Long system life cycles to protect their investment.

- A totally systematic design so that all modules within the software had the same basic "look and feel", making it easier for users to learn and apply the software to their application needs.

0

An architectural design to their automation software, to provide greater flexibility in matching operation requirements across a wide range of applications.

In summary, users require solutions which are modular and functionally independent yet offer a unifying architecture to retain the simplicity of operation offered by the monolithic packages.

\section{Functional Software Architectures}

A totally new software design is emerging for automation systems based on overcoming the constraints of the "packaged" approach, and meeting the requirements of automation users. The new class of soffware resulting from this design approach is referred to as Functional Sofnware Architectures. In this design approach, the architecture itself is the product Paragon INT from Intec Controls is the first member of this new class of software.

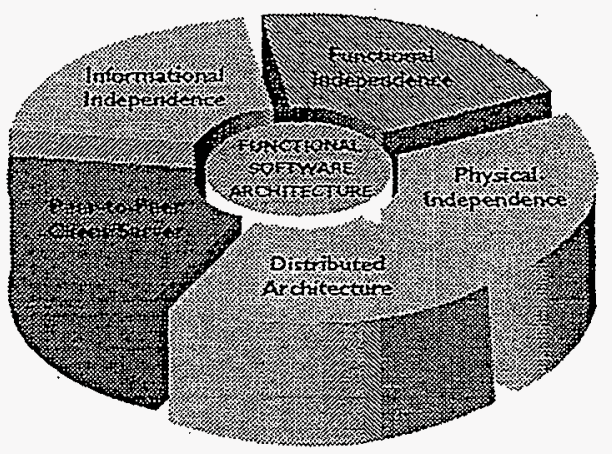


In order to overcome the constraints of traditional software packages, Functional Software Architectures must exhibit several key characteristics. The first major characteristic of Functional Software Architectures is that they are designed around a peer-to-peer client/server computing model. This means that each major automation function is designed to be either a server, a software module that generates, stores and provides information, or a client, a software module that uses and presents information. Each client and/or server has the capability to effectively interact with every other client and/or server in a user-oriented manner. The peer-to-peer aspect of this model means that communications between clients and servers is direct, not via a central server or other type of indirect agent. In many packages that have been said to be based on the client/server model, clients have assumed the active role in the system with servers only taking on a passive data storage role. In Functional Software Architectures, both clients and servers can be active and proactively perfom automation and information management functions. For example, the Control Strategy server can monitor alams and trigger shutdown sequences independently of any client. This provides a much higher level of flexibility and a more natural environment to automation software users.

Another key characteristic of Functional Software Architectures is that the software is designed around a truly distributed approach. Each of the clients and/or servers required for the application can reside either within the same hardware platform or in totally separate hardware platforms networked together either in a local area network, a wide area network, or a combination. The interaction between the clients and servers is the same, regardless of whether they are in the same hardware platform or distributed across a network. The combination of peer-to-peer client/server design and a distributed architecture leads to both effective functional distribution and geographic distribution, so the automation system architecture can be designed around exact automation requirements without sacrificing the ease of use automation users have come to expect.

Functional independence is another key characteristic of Functional Software Architectures. Functional independence means that each software module in the architecture contains all of the necessary functionality to effectively perform its task, either independently or as an integral part of a total automation architecture. Because each module is designed for a specific task, there are no design compromises. The distributed nature of Functional Sottware Architectures applies to identical functional modules as well as different functional modules. For example, if a user needs control in two different locations in the operation, a control server can be put in each location, along with other clients and servers as required. The resuiting architecture operates as a single system. This characteristic allows users to add clients and servers as required without impacting the operating system and provides a high level of location independence across the sysiem. With this characteristic, many of the constraints of the traditional package approach become non-issues.

Physical independence throughout the system is another key characteristic of Functional Software Architectures. Physical independence decomposes into four key subcharacteristics: processor independent design, VO independent design, operating system independent design and communications independent design. Processor independent design means that the sofware modules are not tied to a single processor or processor family, but can run on a wide range of processors, eliminating some traditional processor constraints from the software design. VO independent design means that the software architecture can effecuvely interact with process equipnent through a wide range of $\mathrm{JO}$, and is not limited to a single vendor or small number of vendors $\mathrm{V} O \mathrm{O}$ : Operating system independent design means that the software architecture does not rely 
on the services of a particular operating system, and therefore has the ability to operate on a.variety of operating systems. Communication independent design means that the software architecture is independent of communication protocols and can, therefore, use or interact with a variety of communication systems, both standard and non-standard. This combination of characteristics that comprise physical independence provides automation users with great choice and flexibility while overcoming many of the limitations of traditional packaged systems.

The final characteristic of Functional Software Architectures is information independence. This characteristic provides user-oriented access to all critical information throughout the architecture, independent of access point or information location. The basis for information independence is global object access across the entire architecture. This provides global data point access anywhere in the architecture merely by specifying the name of the data point without requiring network node names or hardware addresses. No knowledge of the network topology is required to effectively operate the system. If you cannot recall a name, the software architecture provides a name browsing facility to help out Because of this global object access, no duplication of data is required throughout the architecture. Data is always stored at its point of origin, and is globally accessible from that point. Information independence also implies that the sof tware architecture operates on uniform data types. For example, real-time and historical data are stored using identical storage methods, allowing for common data usage in rrending and reporting functions. Information independence also applies to file-based data by the incorporation of standard database access techniques such as SQL. This characteristic also supports flexible information consuructs such as CSV, DDE, Networked DDE and even National Language Suppor.

These five characteristics of Functional Software Aschitectures combine to provide a new paradign for automation software that overcomes the limitations of traditional software packages and pave the way for the automation approaches of the next century.

\section{Paragon TNT -- The World's First Functional Software Architecture}

Paragon INT was designed as the world's first Functional Soltware Architecture for automation systems. Paragon TNT incorporates all five Functional Sortware Architecture characteristics into its design, and is clearly modularized into a distributed, functional clienvserver approach. Currenuly, Paragon TNT offers three functionallybased server modules: the Process V/O (PIO) server, Control Strategy (CS) server and Data Manager (DM) server. Paragon TNT also offers a number of client modules: the Operating Interface (OI) clienc, Dynamic Data Exchange (DDE) client, Recipe Management client, Engineering Interface (EI) client and Report client: In addition, the Client Development Kit (CDK) allows users to develop their own clients. The Functional Software Architecrure called Paragon TNT is poised to revolutionize the design of automation systems.

Some industry consultants have agreed that this architecture will be the basis of future automation systens, but predicited that these systems will not become available until 1997. Paragon INT is available now and has been operating in user sites for since mid 1993. You do not have to wait. You can realize the benetits a Functional Software Architecture roday. 


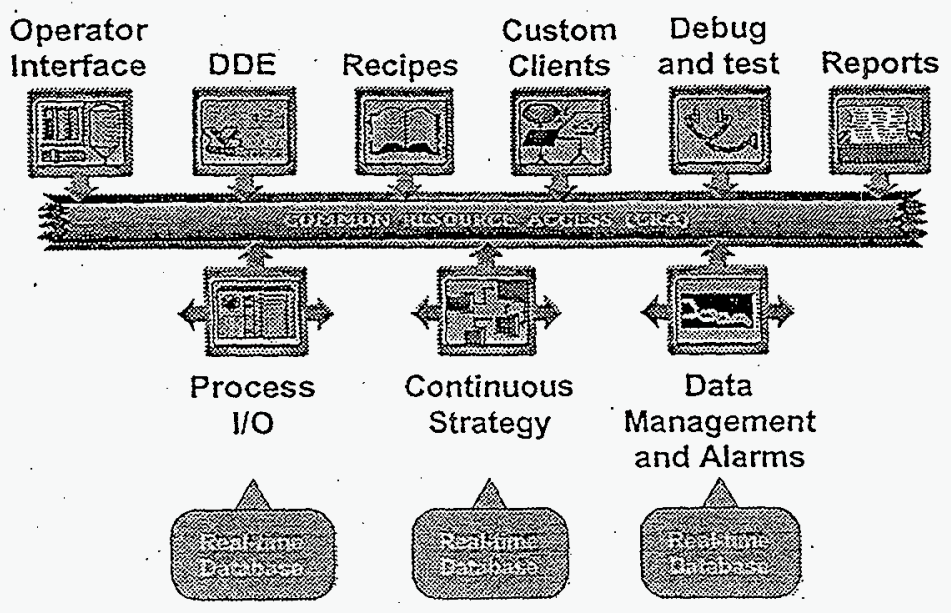

Paragon TNT's Funcrional Software Architecture

\section{The Benefits of Functional Software Architectures}

Merely by describing the chief characteristics of Functional Software. Architectures, many benefits of such an automation systern design are readily apparent. The primary benefits offered by a Functional Software Architecture can be catagorized into five areas, each of which is very important to users as they move forward with their automation strategies.

The first key benefit is the scalability of the software offering. Because of the Functional Software Architecture tesign, users are able to scale the sof tware to exactly meet the requirements of their application, no matter how simple or complex the application may be. No longer must users "force-fit" an ill-suited software package on top of their application. With a Functional Software Architecture, the solution always matches the reguirement on a function-by-function basis.

The second general benefit of Functional Software Architectures is expandability. Expandability is the capability to incrementally add functional components (clients or servers) as your automation requirements dictate and your financial requirements allow. A user can start very small and incrementally grow to an integrated plant-wide automation architecture. Automation components already installed are not impacted on the addition of a function or set of functions.

A very high level of system reliability is the chird of the five key benefit areas incurred by using a Functional Software Architecture. Distribution and independence of function leads to high levels of reliability. Any single fault involves, at most, the 
function in which the fault originates, even when multiple functions are running in the same machine. Also, the modular approach to system implementation, design and test leads to a higher reliability and faster implementation of automation applications.

The fourth key benefit area of Functional Software Architectures is improved system and operational performance. No longer will the limitations of a single hardware platform limit your automation capability. With a Functional Software Architecture, such as Paragon TNT, if you run into a hardware limit, you can either change platforms or add hardware platforms to the architecture without impacting the running modules. Because each functional module contains all resources required to execute its task, valuable CPU resources are optimized since the traditional resource allocation load is reduced. Also, the capability inherent in a Functional Software Architecture typically calls for higher performance software approaches, such as using native 32-bit operating systems as is the case with Paragon TNT, which serves to increase overall system perfomance. If the system is performing optimally, it will clearly contribute to the performance of your entire operation. This is the bottom line for any automation system.

The final key benefit area of Functional Software Architectures is, with a minimal investment, an automation user can maximize the financial return. Users need only purchase the components of the architecture required to meet current needs, and can thereby limit the investment with a "pay-as-you-go" approach that allows users to avoid large up front payments. The Functional Software Architecture provides an easy to use, high performance and functionally focused approach to automation that will minimize demand on critical plant resources, while optimizing the automation through an exact fit of customer automation needs and advance automation techniques. The investment will be small compared to the pay back realized through the use of a Functional Software Archilecture to neet your automation needs.

\section{The Challenge}

The next time you are in the position of deciding on the approprate automation approach for your facility, consider a Funcional Software Architecture. If you believe you could reap the benefits of such an architecture, call intec Controls and ask about Paragon TNT -- the world's first and only Functional Software Architecture for automation. 
InTech

May, 1996

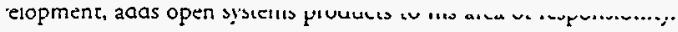

\section{Major automation software upgrades bow}

Arch-rivals Wonderware, Intellution, and USData, plus statistical process control (SPC) sofnvare supplier Gensym,

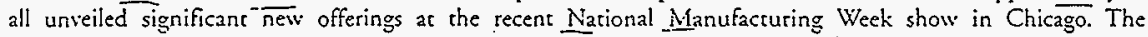
Wonderware Facrory Suire for concinuous, diserete and batch applications is designed to run on Microsofi's BackOffice family of server products, including the SQL Server, which now can be incorporated into InTrack; Windows NT Server; and Microsoft Systems Management Server, as well as the Windows NT Workstation and Windows 95 operating system. Meanwhile, Intellution introduced version 6.0 of irs FIX BOS (batch operating supervisor) system for Windows NT. FIX BOS 6.0 is an open development, process control, and batch management system based on the ISA SP88 batch standard philosophy. The product also provides manufacturing execution system (MES) functions. USData, as expecred (see March In Tech, p. 9), released its FactoryLink Enterprise Control System, which focuses on ease-of-use. Gensym Corp. introduced GSPC, an add-on module to Gensym's G2 Diagnoscic Assistant, to speed configuring online staristical process control applications. 
Design Enginearing

June, 1996

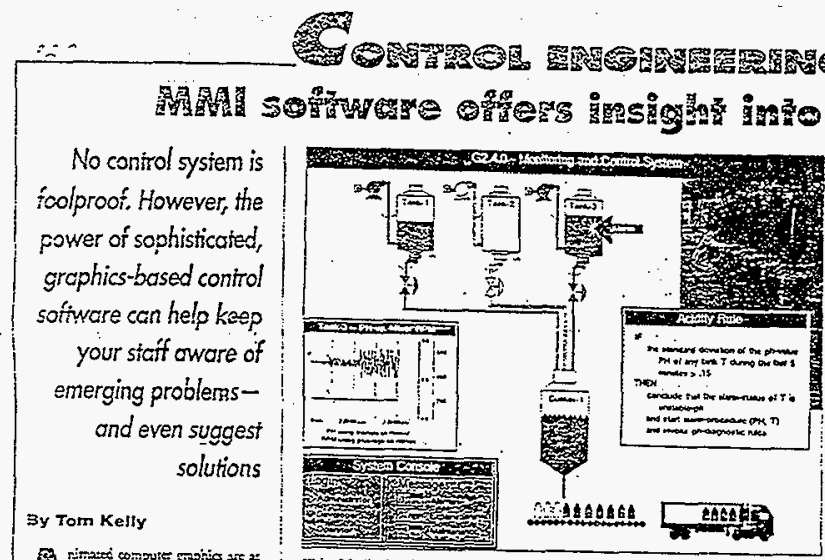

67. rimaves compure frophis are 20 Fye the ber of the mox signifen

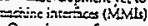

Cre tie pis few yest a ourfoes of companies hove developed softwze which appies anirared granic dirstrys so joctustial control $A$ significas

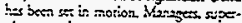
visors and opcestors are pricirs 23

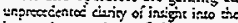
inisras opestions of theif piztrs. reachines ane process

Minimize error

Tats a sinoiogy offess a numide of bes its.

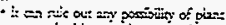

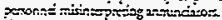
$\because$ hips opernoss recognize whes:

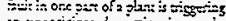

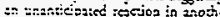
e:-2s tepperec is the Thros Wife is-2s hepen

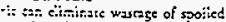
pioeitcr. by continzzily 2tajyzing

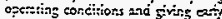
2E-ming wines a procuction line ot:

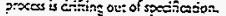

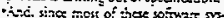
iens =ee jased os Viczoso: "incaws, they can comntriezte wh ethe conpter. to cowntosd 7:0ess tocipes os control secuente tom cheoves or to foud producion

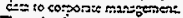

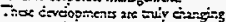
the inive of sipevision and contol

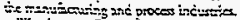
"Ooncenvere Cop. of Invias C.ES wa

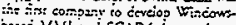
buee XINI and SCADA (eupervisory

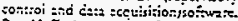

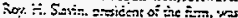

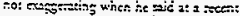
pros-ee introguction: -Sofwes : =

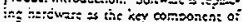

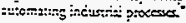

Upsrade cxisting controls

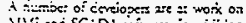

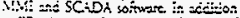

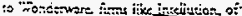
Vo:wood. X́ass, Bovior Adustin इoituze, Eemontor. Gerym Corp..

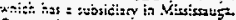

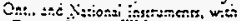

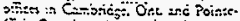
Ci: Quen tave crrcioxis procker Suet actores are compitibie with

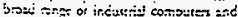

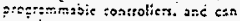

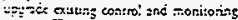

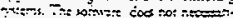

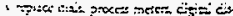

This G2 display from Gentym thows a chenticatorixing process A pernet on the

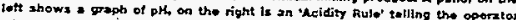
whit to do under probonged devistion of the $\mathrm{PH}$ valus. Teg right is a videa irom a TV camera on the plant troce

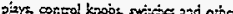
corventional operto istíf: covica

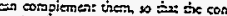

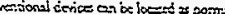

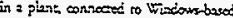
conputses is 2 onseri consoi toom

la many apolicstions. however Fincows-bucé compuien ar loces troughout = plant to give =0p- Rioo: pe

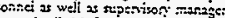
more desied insomentios the is pow

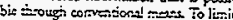
de town of pesing xesed for oxe-

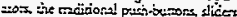
and othe: manua! costois the

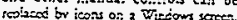

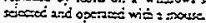

Dynamic displays

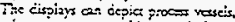

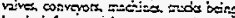
lozced from a chure-uer piece of

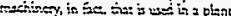
along with all the pipir. clectic

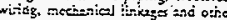

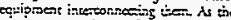

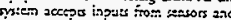
controliets on the oles: os methiner undet control, the animate grophic firow ise moverems tixing pises, sud 2 leveis in vese $x$ miteil bing inaced into = 200

The dispisy an induce expicions of

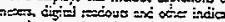
on thowng the values of the vetiobic

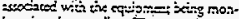

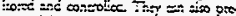

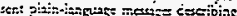
the openeng concitions of frocese of

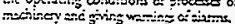

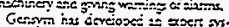
Gerym has brreoper a aper ors icts wich entarce this eprbility oy opersore, witich is epersir sencieid

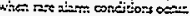

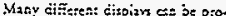

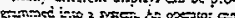

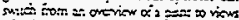

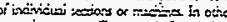

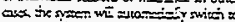

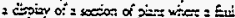
tax 0 -

All this may sounc complez-and

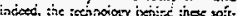
ware peciage is 3 it ti covosers luve

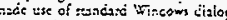
boxa anc coois to wass = essinger in

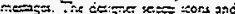

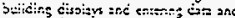

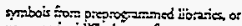

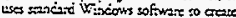

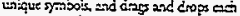
one co move the inco vation

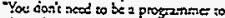
sed. uncisand of troots 25 mpitation," sys Gessyt: of is expert sysen.

Design principles

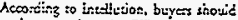
make surc that the Hincows-based

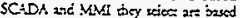

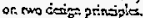

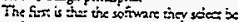
inily compribic with othet types of

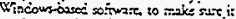
an be upgracod to tew verions sices for zujuys

The soonc principe is thet dor somi-

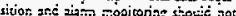
Le inceristed winc you ate perioming rovine Wircous asks, such as opering nles or printing grapitic Invelketon wes

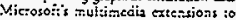
Wiscons so cesere that disping of cu

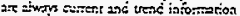
is ziways up-io-dett snd cortect

Statistical softwar

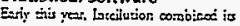

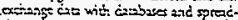

FD SCADNASM

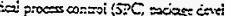

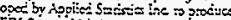

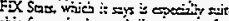

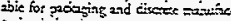
ruring incuestices.

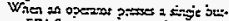
ton. FDX Sars procces retfrom the S.C anc historid $\mathrm{c}_{2}=\mathrm{frer}$

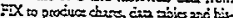
rograns aliowing up ro 225 vetijis $5=0$ as 0.0000

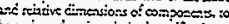

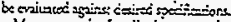

Wore recenty, instiution up 4 ted sofinuare designec to manage bz:en oroceses througinoul 27 etieprie : $=\mathrm{DX}$ SOS 6.0 (Esté Ope-sins Seperixor)

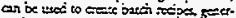

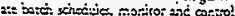
buch prodineion provic: ral-5isc din

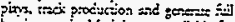

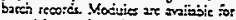

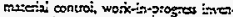

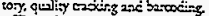

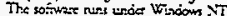

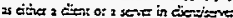

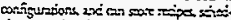

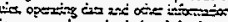
in commoriy-used reiztonal cisinst Mitoosit SQL Seve Osox zed Subre

One wiacow of $2=$ fried an displas $y$ live viceo fom teis conces insolict Srrice Number $3 / 3$

Managementsottware

Diring the Xarioal Yenézerutes

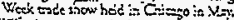
Wondenser urveliec a prodiue stich

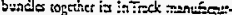
ins exection sytich ( $M E S$ ) with ing exection sytic

inTrek sreds work-in-profas ase

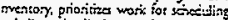

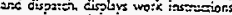
25 cach munúfersing sep. mositor

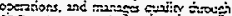
SPC 2nd anzivsis of solitend di-

is is the tis of a seic of prof in

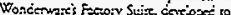

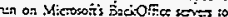

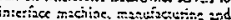
proses: control to comorrictiene. sys

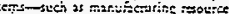

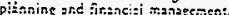
Reader Serit Nurredr jit2

Expert systems

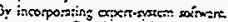
known as C2, h:o Woncenzats la Toued MY! sofwate Getsry as

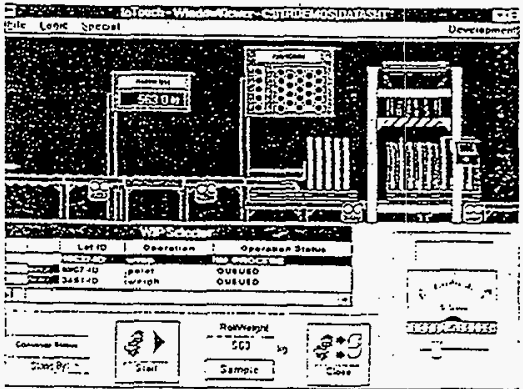

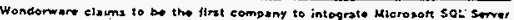

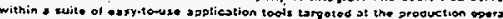
tions of a venety of incustriet.
$H N F=1539$ 


$$
300+\frac{1}{1}
$$

exostried the informetion tar con be

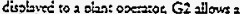
isifice to progers i concral or superisory eomputer so follow the wene ruis

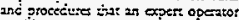

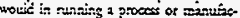
turizs opersion Thex rules en be prith

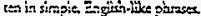

lasted of sisoly showiog an slarm. G2 can wase operators ez-ly in: a

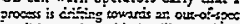
condition and provide inteligent advies on how to wixe colrective tesion," spy

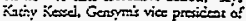
n-xixitis.

As weil us mosizoring and disgnosis.

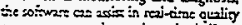
managtrets, eymamie rcieduling,

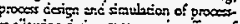

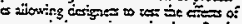
proposed cings in producion Redir Srike Nenter sil

Production scheduling

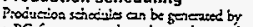
a PC frors up-ro-the-mintute c2s 2ad infor-2xion from 2 simp fios oric desi

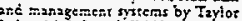
industriai So.twert's TESS sebeduting preiser

TEŚS mositor protucion and itigh. ligins probiens rud is machixe domtithe of !zet of :esources. Is provide tithe of leck of :eources. If provide

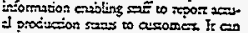
il phoivesion rass w assomex Ir con

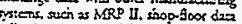
collecrion strens and depboce

Desigaers fanitiar with rejey-lacicer logic protramming of PLCs will find

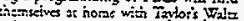

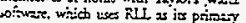

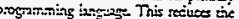

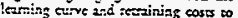

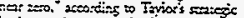

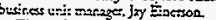

The soitwarc tiso irtegranes with Wirciows appiletions and allows orogrems to be writen in ClCt+. Visual Basic and other begrages Sexsions en in concirenty os cis ladide logie en

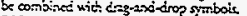

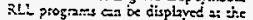

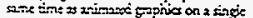
se:cen. The sofwase or cire mixed

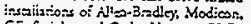
$C \equiv$ Seripie and Caje Himme PLC

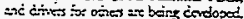
Ratim Sorser Nivmor 310

\section{Easy programming}

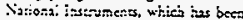

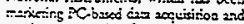

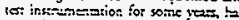

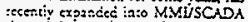
througn is accuisition of Gcorgetionn Sysicns. ceveloper of a syrear elled Lopiose

Nationat testomed it to a 32-bi:

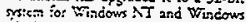

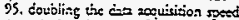
A paricin acivanese of Lookout is tha it =laxs a deigne to improve or upgrade

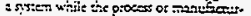
in ine wece contoi is riming PLC Inc licic ito connesions, grophics 27

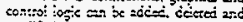

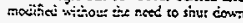
1 Froxes.

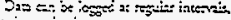
on $\varepsilon$ er

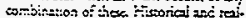
ime duts ate presented in a tetaice

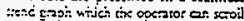

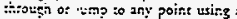

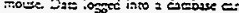
be tec itte of se appitetions - is 1prescines Vimores 509

\section{Trent to openness}

That mox: trent arreiopment oxenpiif : : and incustrial control-an ioctessiag opennes and compatiolizy witi otice

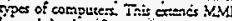
byoné tiv shop foor in proses noto

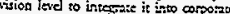
iniormation syetent Shop sos - med proces menages can now hive a soper ot evey puice is a plars no mette bow complex And serios stinagenent can be givea the ability to make the swif chinges to production nexersy to be tale to repond to socizy's mpid ester فेxา

Tom Kally is Tecbnicai Fiteld Edtor Eleoronic

Laokout, trom Nations Invtromats, Is in fully contlguesste, objinct oriont ad, event-driven Mest ane ScAa Dalkage for Windows os and $\mathrm{KT}$.

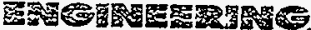
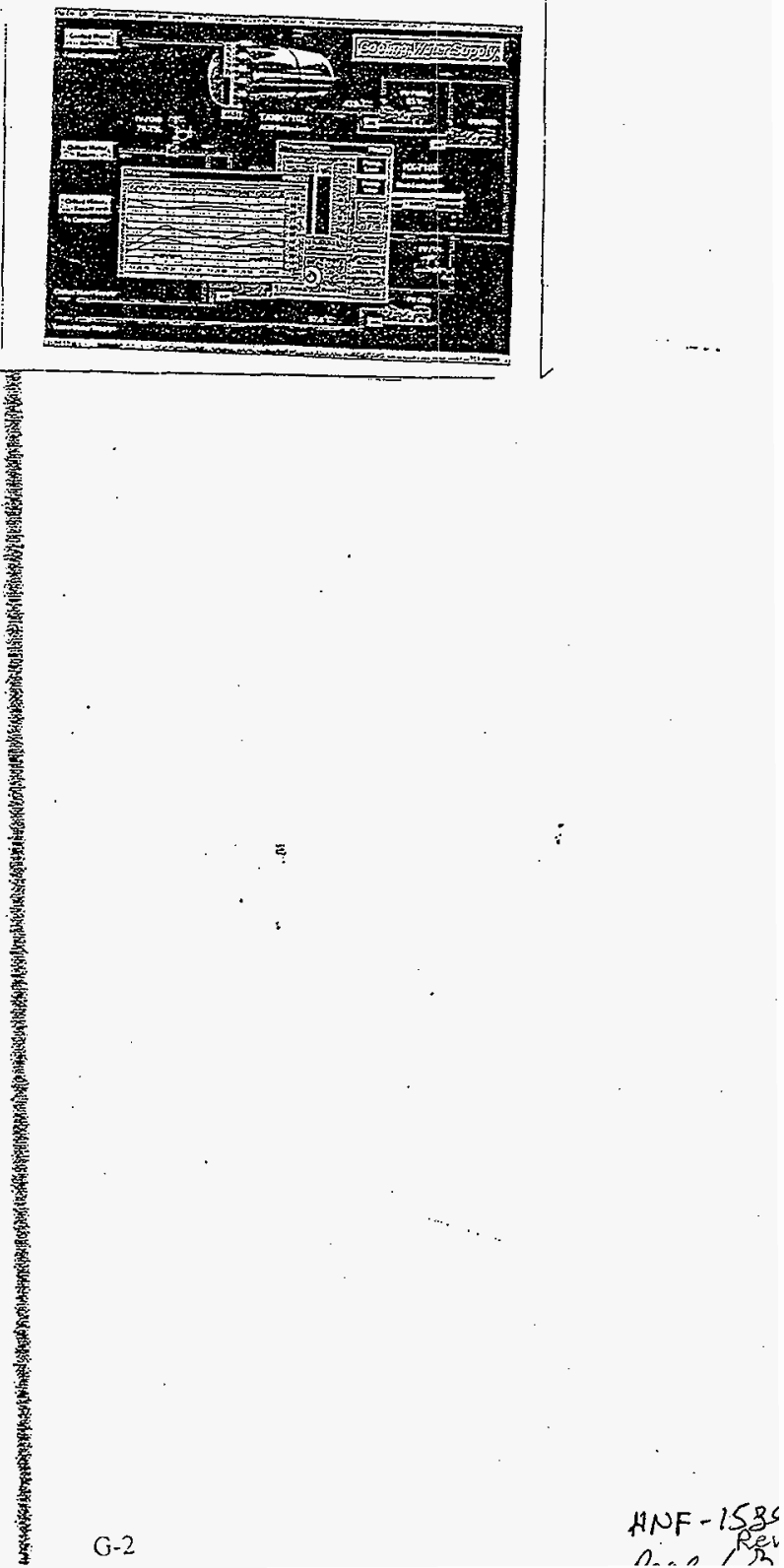


\section{Have Reports of the Death of the Greatly Exaggerated?}

D eskitop and industrial PCs are perruesting the factory floor in increasing numbers. The operatorinterface $(O / D)$ task, once the domain of the industrial workstation, is being performed with graphical brilliance by the PC. When eramining the market and technical forces at work in the operator-interface market, one concludes that reports of the death of the industrial workstation have been greatly exsgrerated.

Let's first examine the forces and trends that brought the PC to the faictory floor.

1. $\mathrm{PCs}$ are cheap with respect to their considerable computing power. They excel at displaying and storing data and connecting to other computers.

2. Enabling the use of PCs in industrial environments has been the explosion of Wiadows. based $O / 1$ software applications (i.e., Wonderware, Intellution and USdata). These packages give the user easy means to create rich graphical, animated operator-interface screens.

3. Windows-based OII software brought unprecedented connectivity to all brands of PLCs and remote HO networks, and to plant computing networks like Ethernet and TCP/TP. It also brought software connectivity that allowed several programs to run simultaneously and share data.
Now let's examine the forces that limit the PC as an operator interface in industrial applications.

1. The desktop $P C$ is packaged for the desktop. A large percentage of control-systems designers opt for industrially packaging PCs. Prices for industrial PCs start at more than twice that of desitop PCs.

2. The Windows-based $O / I$ sofware applications are expensive Unlimited "tag" development systems list for $\$ 5000$, individual runtime licenses go for $\$ 1500$, and PLC connection drivers command $\$ 250$ to $\$ 500$.

3. Windows-based run-time systems are resource hogs. If you are running Windows you need at least a 486 at $66 \mathrm{MFz}$ with $8 \mathrm{MBB}$ of memory and a big hard disk. Unfortunately, memory prices have been stubbornly ixed due to shortages

The PC as an operator interface has extensive capabilities, and these capabilities carry a price. This price is in many instances at odds with another market trend - the growth of mini and micro PLCs. These are extremely capable, range in price from $\$ 500$ to $\$ 2500$, and are the fastest growing segment of the PLC market.

How can the industrial workstation cornpete with the venera ble PC? The general and open architecture of the $P C$ and its software let it be all things to all users. The new industrial workstation will find new life by being all things to some users. But how can it compete with bit-mapped graphics and animation, industry-standard expansion, and connectivity to major P.LCs?

\section{THE NEW HARDWARE}

The new workstation will effec. tively be a PC - not a full-blown PC, but a-mini-PC or mutant-PC or an embedded $P C$, one stripped of its excess baggage and optimized for workstation functionality. This PC will use an Intel code compatible processor such as the $25 \mathrm{MHz} 80386 \mathrm{EX}$ processor This highly integrated processor and chipset family brings the benefits of the PC to the workstation, such as local bus graphical horsepower for detailed graphics and object animation. This approach also gives the rorkstation the same industry-standard expansion and connectivity options as the PC via an ISA bus, serial ports, parallel port and PC-card expansion.

For economy and ruggedness, the PC's hard drive is discarded in favor of flash memory. Expensive DRAM inemory will be minimized by shunring the Windows environment. High-resolution color flat-panel displays will facilitate compact, rugged packaging and effective display of graphical objects. 
$\exists=-\because \ldots$

\section{Industrial Workstation Been}

\section{THE NEW SOFTWARE}

The new workstation user demands dragging and dropping of graphical objects like pushbuttons, pilot lights, gauges and bar graphs. For ease of screen development, nothing beats a Windows-based development system. What about the run-time system? Windows? No, because it's a resource hog. How about a small real-time execuitive that provides real-time response, great graphics, and requires $1 / 16$ the memory and no hard disk? This is the new worisstation paradigm: develop on your desitop Windows system and download to the flash memory of a robust, real-time, PC-compatible engine. As far as pricing goes, workstation development systems have traditionally cost $\$ 500$ to $\$ 1500$, and run-time licenses are usually free.

Where will this new worlesta. tion and a home amidst the PC explosion? The answer is $O / I$ applications of low-to mid-range complexity. As a general rule, if a wor Lstation meets your needs, it will be a lower-cost solution than any PC solution. Using the following guidelines, you should be able to determine whether you are a good candidate for a PC or a woristation.

1. If your $O / I$ task will require a dispiay of larger than 14", buy a DC. Displays are expensive, and woristations with larger cisplays cost as much as PCs.
If your $O / /$ screens are so detailed that they need large, high-resolution displays, the application probably needs a $\mathrm{PC}$ to run.

2. Analyze your $\mathrm{O} / \mathrm{I}$ task. If you will primarily be displaying screens consisting of pushbuttons, indicator lights, gauges and alphenumeric text, a workstation should be fine. Even if you venture to object animation, alarming, reports and recipes, you should be OK with a workstation. But if you need to display more than a few bit-mapped images, along with more than 50 or 100 screens, you'l tax the limited memory of a workstation. Logging, trending, scripting and simultaneously runaing other programs (i.e., MS-Excel or SPC pactage) are also beyond the capabilities of the workstation.

3. Workstations are designed to connect to popular PLCs. This connection is typically an RS. $232 / 422 / 485$ variant and is built into the workstation. If you need to connect to a PC or other computer(s) via an Etherat. fiber-optic or other high-speed lints, you are in the domain of the PC.

Woristation and $\mathrm{PC}$ technologies, both hardware and software, have merged to create a new breed of workstation that boasts improved expansion. graphics, processing perfor- mance, and ease of use. The merging of techrologies will be of great value to control-system designers faced with low- to midrange $O / I$ applications.

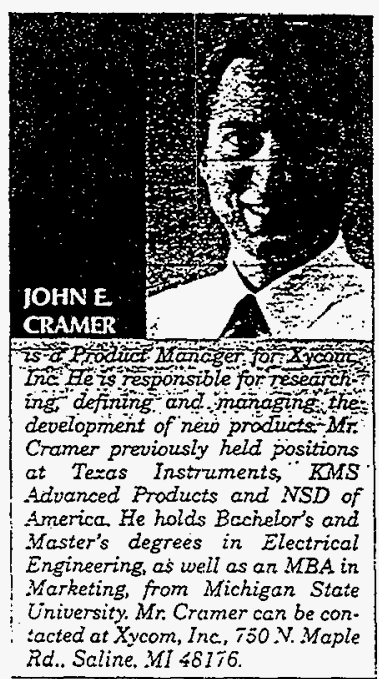




\title{
1,100 \\ $0 \cdot n a y \cdot 6 \varepsilon S 1-\exists N H$
}

S6 smoptes of sion crostryou sol sroputh

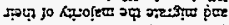
. Hee sol sost sonx or sopers

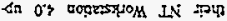

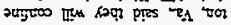

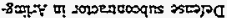

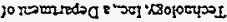
voseryneturos ecopol it for

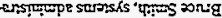

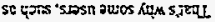

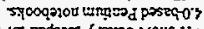

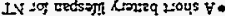

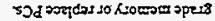

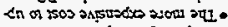
sरem

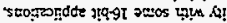

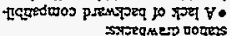
-P्र

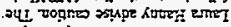

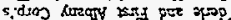

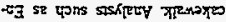
of sq IIM IN का stinotr ing

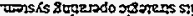

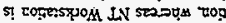

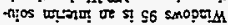

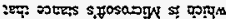

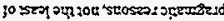

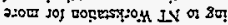

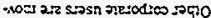
pIEs $04_{4}$ 's7ogezs
-

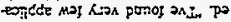

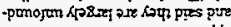

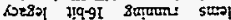
-qod jo sisodas jo porgoss suop

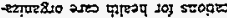

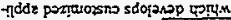

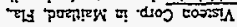

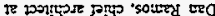
pres रप . . गश००

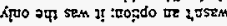

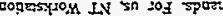

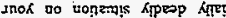

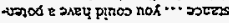
- पI 20J 202025

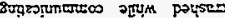
ansis $g 6$ smopgu $\mu$. $\pi_{\text {sew }}$

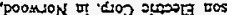
uonersy $20 \mathrm{M}$ iN to scoperydde
$\Rightarrow$ Dर soleted inpotd stessis 8tum

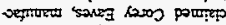

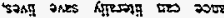

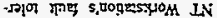

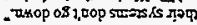

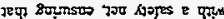
sosn spliojd togaso:oM iN

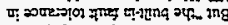

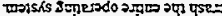

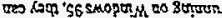

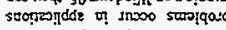

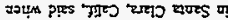
Eno:s torरe

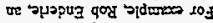
pres siskifer ptre stasn stor

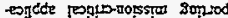

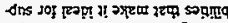

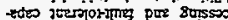

ortapte sexsedpme spip -

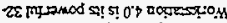
LN sinopum to ग्या 2TC क्षा

प०सद्य | st ing s5 smoptry गe: pres

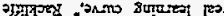

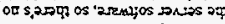

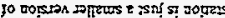

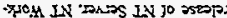

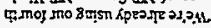

So seopt? $M$

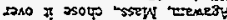

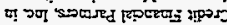

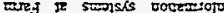

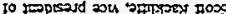

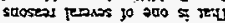

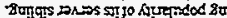

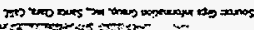

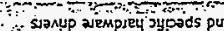

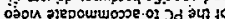

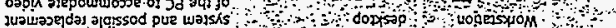
mor.

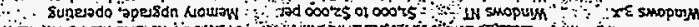

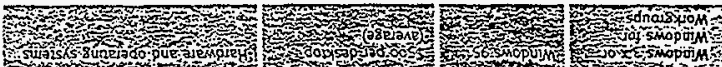

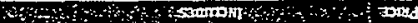

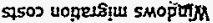

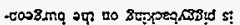

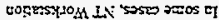
$\operatorname{traximas}$

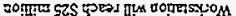

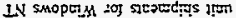

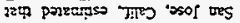

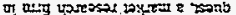

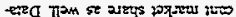

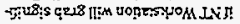
Se Fy

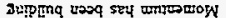

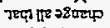

or popordxo s! 07 IN Smopts

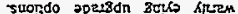

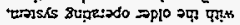

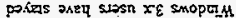

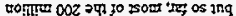

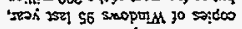

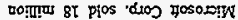

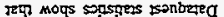
scopisop

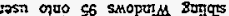

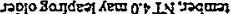

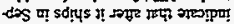

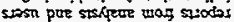

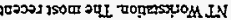
smoptti $M$ poof rongaderos pos

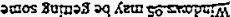

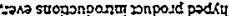

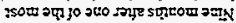

o!c!a wme.$k g$

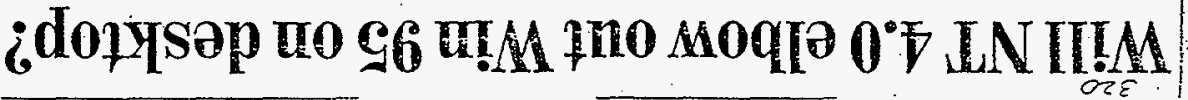

\author{
966I '0Z AEUT \\ plomianduroj
}

